\title{
Simulation of floating structure dynamics in waves by implicit coupling of a fully non-linear potential flow model and a rigid body motion approach
}

\author{
E. Dombre - M. Benoit • D. Violeau • C. Peyrard • \\ S. T. Grilli
}

Received: 29 April 2014 / Accepted: 22 October 2014 / Published online: 13 November 2014

(C) Springer International Publishing AG 2014

\begin{abstract}
We demonstrate the accuracy and convergence of a new numerical model solving wave-structure interactions based on the fully non-linear potential flow (FNPF) theory coupled to a rigid body motion approach. This work extends an earlier model proposed by Guerber et al. (Eng Anal Bound Elements 36(7):1151-1163, 2012), restricted to fully submerged structures, by allowing to solve for freely floating bodies on the free surface. Although we are currently extending the model to three dimensions (3D), the work reported here only considers two-dimensional (2D) problems. We first introduce the FNPF model, originally developed in 2D by Grilli et al. (Eng Anal Bound Elements 6(2):97-107, 1989), Grilli and Subramanya (Comput Mech 17(6):374-391, 1996), and later extended to 3D by Grilli et al. (Int J Numer Methods Fluids 35(7):829-867, 2001). We present the implementation by Guerber et al. (Eng Anal Bound Elements 36(7):1151-1163, 2012) in the 2D-FNPF
\end{abstract}

E. Dombre $(\bowtie) \cdot$ M. Benoit · D. Violeau · C. Peyrard

Saint-Venant Hydraulics Laboratory, Université Paris-Est (EDF

R\&D, ENPC, Cerema), 6 quai Watier, 78400 Chatou, France

e-mail: emmanuel.dombre@edf.fr

M. Benoit

e-mail: michel.benoit@edf.fr

D. Violeau

e-mail: damien.violeau@edf.fr

C. Peyrard

e-mail: christophe.peyrard@edf.fr

S. T. Grilli

Department of Ocean Engineering, University of Rhode Island,

Narragansett, RI 02882, USA

e-mail: grilli@oce.uri.edu model of van Daalen's implicit method for fluid-structure interactions [see van Daalen (Numerical and theoretical studies of water waves and floating bodies, $\mathrm{PhD}$ thesis, Universiteit Twente, The Netherlands 1993) and Tanizawa (Proceedings of 4th Osaka colloquium on seakeeping performance of ships 2000)]. We then detail the numerical scheme used for coupling the FNPF model to the motion of a floating rigid body. Moreover, we propose a new numerical strategy for advancing the free surface front inspired by symplectic integrators, which achieves a much better performance for energy conservation. The developed algorithm is first applied to forced motion cases, for which analytical and experimental results can be found in the literature and used as benchmarks. The accuracy of the numerical solution for the fluid and applied forces is then discussed for cases with small or large amplitude motion. In the latter case, a preliminary investigation of non-linear effects is performed for the classical application of a semi-circular heaving cylinder, by comparing the computed hydrodynamic force to the experimental measurements of Yamashita (J Soc Nav Arch 141:61-70, 1977). In particular, the comparison of the magnitude of the force harmonics, up to the third order, shows the importance of simulating non-linear interactions, which become important as the ratio of the radius of the cylinder over the wavelength increases. In a second set of applications, we assess the model accuracy in dealing with freely floating bodies. As a first test case, we consider the decaying motion of a freely heaving horizontal circular cylinder released from a non-equilibrium position above the free surface. In this more demanding computations, we verify that total energy fluid-plus-body motion is well conserved, which confirms the accuracy of the fluid-structure interaction algorithm. As a second test case, we consider the free motion of a rectangular barge in waves and compute the first-order response amplitude operators. 
Keywords Wave-body interactions - Non-linear wave · Numerical wave tank . Floating bodies $\cdot$ Boundary element method · Wave forces

\section{List of symbols}

$\Omega_{f}(t)$
$\partial \Omega_{f}(t)$
$\Gamma_{f}(t)$
$\Gamma_{b}$
$\Gamma_{l}$
$\Gamma_{c}(t)$
$\mathbf{v}$
$\phi_{t}$
$\mathbf{x}_{f}$
$\mathbf{v}_{b}(\mathbf{x})$
$N_{\mathrm{el}}$
$N_{\mathrm{dof}}$
$N_{\mathrm{b}}$
$O_{k}$
$N_{k}$
$x_{b}(t)$
$p_{a}$
$\ddot{\mathbf{x}}_{G}(t)$
$\dot{\mathbf{x}}_{G}(t)$
${ }_{\mathrm{d}}(t)$
$\mathbf{x}_{G}(t)$

Volume of fluid at time $t$

External boundary of the fluid at time $t$

Set of free surface points at time $t$

Bottom boundary

Set of lateral boundaries of the domain $\Omega_{f}(t)$

Wetted surface of the body boundary at time $t$

Fluid velocity vector

Velocity potential such that $\nabla \phi=\mathbf{v}$

Eulerian time derivative of $\phi, \phi_{t}=\frac{\partial \phi}{\partial t}$

Position vector

Solid velocity vector at the point $\mathbf{x}$

Number of boundary elements

Number of degrees of freedom

Number of degrees of freedom on the body surface

Green's function, fundamental solution of the Laplace equation

Lagrangian time derivation

Position vector of the center of mass of the body

Velocity vector of the center of mass of the body

Acceleration vector of the center of mass of the body

Unit outward normal vector to the fluid boundary $\partial \Omega_{f}(t)$ at point $\mathbf{x}$

Unit inward normal vector to the body boundary $\Gamma_{c}(t)$ at point $\mathbf{x}$

Atmospheric pressure

Set of free surface nodes belonging to $\Gamma_{f}(t) \cap \Gamma_{c}(t)$

Set of body surface nodes belonging to $\Gamma_{f}(t) \cap \Gamma_{c}(t)$

Number of element of the set $\Gamma_{f}(t) \cap \Gamma_{c}(t)$

Function such that $\phi_{f}(t)(\mathbf{x}, t)$ is the Dirichlet condition on the free surface at point $\mathbf{x}$ and at time $t$

Canonical basis of $\mathbb{R}^{3}$ such that $\mathbf{e}_{1}=\mathbf{e}_{x}$, $\mathbf{e}_{2}=\mathbf{e}_{y}$ and $\mathbf{e}_{3}=\mathbf{e}_{z}$

Horizontal axis

Vertical axis

Transverse axis

$k$-th component of the vector $\mathbf{f}, f_{k}=\mathbf{f} \cdot \mathbf{e}_{k}$
$X_{t}$

$\rho$

$\mathbf{F}\left(t_{i}\right)=\mathbf{F}_{i}$

$\mathbf{F}_{i, 0}^{*}$

$a_{i j}$

$b_{i j}$

$c_{n}$

$g$

$\mathbf{F}_{i, k}$
Transformation which maps the initial domain $\Omega_{f}(0)$ on $\Omega_{f}(t)$

Density of the fluid

Hydrodynamical force acting on the body at time $t_{i}$

Prediction of the hydrodynamical force acting on the body at time $t_{i}$ made at iteration $i-1$

Added mass coefficient in the $i$ direction due to a motion in the $j$ direction

Damping coefficient in the $i$ direction due to a motion in the $j$ direction

Fourier coefficient of order $n$

Acceleration of gravity

Hydrodynamical force acting on the body at time $t_{i}$ and sub-iteration $k$ in the Newmark scheme

\section{Introduction}

The numerical modeling of the interactions of floating structures with waves is very challenging, particularly when one wishes to include in the model both the full non-linearity of the free surface boundary conditions as well as large motions of the structure, yielding a possibly highly non-linear behavior of the equations of motion, even using simplified fluid models such as fully non-linear potential flow (FNPF). For a long time, for efficiently predicting both body motion and forces, the engineering design practice has focused on linearizing free surface boundary conditions in the FNPF model as well as assuming small body motions. The use of an inviscid model was typically compensated by introducing a viscous drag force based on semi-empirical formulations such as Morison's equation.

However, the use of such simplified models is often limited to the order-of-magnitude computation of upper bounds of important parameters, such as the steepness and the amplitude of the motion. For the innovative floating structures that are being considered for new applications in ocean renewable energy, such as floating wave energy converters or floating offshore wind turbines, these simplifying hypotheses could significantly affect the eventual design, perhaps making it too sturdy and costly in some cases, or worse unsafe in others.

In the realm of academic research, since the late 1980s, more advanced and complete models such as FNPF have been developed and used, typically based on a higher-order boundary element method (HO-BEM) (e.g., Grilli et al. 1989; Grilli and Subramanya 1996). However, despite a constant increase in computational resources, the coupling of such FNPF models with floating rigid body motion has been mostly studied for two-dimensional (2D) geometry; recent contributions in this respect are based on a BEM solver and a force 
decomposition method (Koo and Kim 2004, 2007; Tang et al. 2011). Other numerical methods were also applied, such as the finite element method (FEM) with an arbitrary Lagrangian Eulerian (ALE) formalism to follow the internal points in a 2D numerical wave tank (Yan and Ma 2007); the latter approach was extended to 3D geometry (Ma and Yan 2009). These authors used a direct approach for evaluating the boundary conditions; by contrast, here, we will use an implicit model, based on the earlier work by Guerber et al. (2012), who implemented van Daalen's (1993) and Tanizawa's (2000) implicit method in Grilli et al.'s 2D-FNPF model (Grilli et al. 1989; Grilli and Subramanya 1996) to study the interactions of a fluid with fully submerged structures.

Some 3D models solving wave-structure interactions for floating rigid bodies have also been proposed, particularly, in the field of naval architecture. See, for instance, the work by Liu et al. (2001) or, more recently, by Wang (2005) who used a desingularized BIE method on unstructured grids. Note that an early attempt at developing a 3D axisymmetric model was proposed by Dommermuth and Yue (1987). Applications with the panel method in forced and free motion of a sphere were also found in the work of Berkvens (1998).

Regarding floating bodies without forward speed, Bai and Eatock Taylor (2006) studied an oscillating cylinder as well as a bottom mounted cylinder using a HO-BEM. Later, these authors also considered the case of flared structures for bottom mounted and truncated cylinder in Bai and Eatock Taylor (2009), using an auxiliary method for coupling the fluid and the solid. The different modes of body motion were more specifically studied in a recent paper by Zhou et al. (2013). For completeness, numerical models based on the full Navier-Stokes equations were also proposed in 2D (Rahman et al. (2006); Hadžić et al. (2005); Ghasemi et al. (2014)) and, in particular, wave impact on structures in regular and irregular waves was also investigated in $\mathrm{Li}$ and $\mathrm{Lin}$ (2012). But the accurate direct solution of Navier-Stokes equations for non-linear wave-structure interaction problems, particularly in 3D, still remains a computationally formidable task that poses greater numerical difficulties than when using potential flow theory.

Here, we study wave-structure interactions for floating rigid bodies, using a FNPF model, by extending the work of Guerber et al. (2012), who studied freely moving submerged rigid structures. In the latter work, it was observed that, when the submerged body moved close to the free surface, numerical problems occurred that reduced the model convergence and accuracy and, hence required special attention in the numerical implementation. In this paper, we will investigate whether similar problems also occur when the floating body is attached to the free surface. Although this paper is limited to $2 \mathrm{D}$ problems, this work is a preliminary step since our main goal is to develop this method in $3 \mathrm{D}$, based on the 3D-FNPF model by Grilli et al. (2001).

As compared to earlier methods discussed above, the present model relies on a fully Lagrangian scheme for updating both the free surface and the body geometry. To ensure accuracy in the BEM, at each time step, free surface discretization nodes of the FNPF model are regridded to equal spacing (curvilinear in 2D). As observed in earlier applications of this 2D-FNPF model, the high accuracy and stability of numerical results make it possible computing the body motion for a long time, without experiencing instabilities that would otherwise require smoothing, as done in most other similar models (e.g., Koo and Kim 2004). Moreover, the fluid-structure coupling through the hydrodynamic force, is based on a fully implicit scheme. The use of the auxiliary method was found in Koo and Kim (2004), and a direct evaluation of the dynamic boundary condition on the solid was found in Yan and Ma (2007). For the case of the forced heaving motion of a 2D horizontal cylinder, results of our model are compared with the experiments of Yamashita (1977).

The paper is organized as follows. In Sect. 2, we present the model governing equations and their numerical implementation. Then, we present in Sect. 3 the comparison of numerical results to linear, weakly non-linear, as well as experimental results, for the forced heaving motion of a $2 \mathrm{D}$ cylinder. The special interest of having a fully non-linear model will be outlined. The sway motion of a rectangular body is addressed in Sect. 4. In Sect. 5, we study the model convergence properties for free decay tests of a floating circular cylinder, for which a semi-analytical solution due to Maskell and Ursell (1970) is available. Many numerical and physical parameters will be varied, to assess the sensitivity of the model solution. Finally, we assess in Sect. 6 the capability of the model to predict the free motion of a rectangular barge in waves.

\section{Governing equations}

In the following, we use notations and definitions introduced in earlier publications of the 2D-FNPF model (Grilli et al. 1989; Grilli and Subramanya 1996; Guerber et al. 2012; see also the definition section). Thus, the boundary of the fluid domain $\partial \Omega_{f}(t)$ is divided into four parts, on which different types of boundary conditions can be applied, $\partial \Omega_{f}(t)=\Gamma_{f}(t) \cup \Gamma_{c}(t) \cup \Gamma_{b} \cup \Gamma_{l}(t)$, with $\Gamma_{f}(t)$ denoting the free surface, $\Gamma_{c}(t)$ the rigid body surface, $\Gamma_{b}$ the bottom surface and $\Gamma_{l}(t)$ the lateral boundaries. The bottom boundary $\Gamma_{b}$ is assumed to be time independent.

\subsection{Mathematical model and assumptions}

We assume the fluid to be incompressible and inviscid and the flow to be irrotational. This ensures the existence of a 
scalar velocity potential $\phi$, such that the flow velocity is, $\mathbf{v}=\nabla \phi$. In all cases, the potential $\phi$ must satisfy mass conservation, expressed as a Laplace equation within the entire fluid domain $\Omega_{f}(t)$. Moreover, by considering the successive time derivatives of this equation (denoted by a superscript), it is easily seen that $\phi^{(k)}(k=1, \ldots)$ is also harmonic.

Denoting by $G(\mathbf{x}, \mathbf{y})=-\frac{1}{2 \pi} \log (\|\mathbf{x}-\mathbf{y}\|)$ the fundamental solution of the Laplace equation in $2 \mathrm{D}$ (i.e., $\mathbb{R}^{2}$ ), and applying Green's divergence theorem (see e.g., Grilli and Subramanya 1993; Bonnet 1995), the Laplace equation is recast as a Fredholm equation of the second kind:

$$
\begin{aligned}
& c(\mathbf{x}, t) \phi(\mathbf{x}, t) \\
& \quad=\int_{\left.\partial \Omega_{f}(t)\right)}\left[\frac{\partial \phi}{\partial n}(\mathbf{y}, t) G(\mathbf{x}, \mathbf{y})-\phi(\mathbf{y}, t) \frac{\partial G}{\partial n}(\mathbf{x}, \mathbf{y})\right] \mathrm{d} S_{y}
\end{aligned}
$$

where the function $c(\mathbf{x}, t)$ denotes the fraction solid angle seen from the boundary point $\mathbf{x}$, e.g., for a regular portion of the boundary $\partial \Omega_{f}(t)$, we have $c(\mathbf{x}, t)=\frac{1}{2}$. This BIE is amenable to a numerical solution by a BEM.

Two boundary conditions apply at the (time varying) free surface. The first one expresses the continuity of the pressure at the free surface $p=p_{a}$ on $\Gamma_{f}(t), p_{a}$ being the atmospheric pressure, which is known as the dynamic free surface boundary condition. Additionally, a second boundary condition, the kinematic free surface boundary condition, must also be satisfied on $\Gamma_{f}(t)$, which states $\frac{\mathrm{d} \mathbf{x}}{\mathrm{d} t}=\nabla \phi$. The latter condition expresses that water particles belonging to $\Gamma_{f}(t=0)$ remain on $\Gamma_{f}(t)$ for any given time $t>0$.

On the solid boundary of the floating body, $\Gamma_{c}(t)$, we specify a free-slip condition, which expresses the normal derivative of the potential, equal to the normal fluid velocity as:

$\phi_{n}(\mathbf{x}, t)=\frac{\partial \phi}{\partial n}(\mathbf{x}, t)=\mathbf{v}_{b}(\mathbf{x}, t) \cdot \mathbf{n}_{b}(\mathbf{x}, t)$

where $\mathbf{n}_{b}(\mathbf{x}, t)$ denotes the unit normal vector pointing inward to the solid surface $\Gamma_{c}(t)$, at point $\mathbf{x}, \mathbf{v}_{b}(\mathbf{x}, t)=\dot{\mathbf{x}}$ is the body velocity, with the upper dot denoting the Lagrangian time derivative following the body motion, $\frac{\mathrm{d} \text {. }}{\mathrm{d} t}$ and $\cdot$ denotes the usual Euclidian scalar product. This condition remains valid on the fixed bottom and lateral boundaries, $\Gamma_{b} \cup \Gamma_{l}$, using a zero velocity, i.e., $\frac{\partial \phi}{\partial n}=0$.

The above set of equations can be recast as the following boundary value problem:

Find $\phi$ such that:

$$
\begin{aligned}
& c(\mathbf{x}, t) \phi(\mathbf{x}, t) \\
& \quad=\int_{\partial \Omega_{f}(t)}\left[\phi_{n}(\mathbf{y}, t) G(\mathbf{y}, \mathbf{x})-\phi(\mathbf{y}, t) \frac{\partial G}{\partial n}(\mathbf{y}, \mathbf{x})\right] \mathrm{d} S_{y} \\
& \phi=\phi_{f}(t) \text { on } \Gamma_{f}(t) \\
& \phi_{n}=0 \text { on } \Gamma_{b} \text { and } \Gamma_{l} \\
& \phi_{n}=\dot{\mathbf{x}} \cdot \mathbf{n} \text { on } \Gamma_{c}(t)
\end{aligned}
$$

$\phi_{f}(t)$ is the value of the potential on the free surface $\Gamma_{f}(t)$, whose geometry and kinematics are assumed to be known at initial time $t=0$. For later time, these are computed in the numerical model, using the time-stepping scheme presented in Sect. 2.2.

As reported by many authors, the use of a finite difference scheme for evaluating the time derivative of the potential tends to be numerically unstable (Koo and Kim 2004; Yan and Ma 2007). Following Guerber et al. (2012), instead, we apply the same BIE technique for computing $\phi_{t}$, which satisfies the same field equations. This function has no direct physical interpretation unlike the acceleration potential $\phi_{t}+\frac{1}{2} \nabla \phi \cdot \nabla \phi$, whose gradient is equal to the fluid acceleration $\frac{\mathrm{d} \mathbf{v}}{\mathrm{d} t}$ as pointed out by Tanizawa (1995). However, it has the advantage to be also solution of Laplace equation, which makes it computationally efficient to solve. Besides, in the time stepping schemes used here (see Sect. 2.2), we need to compute $\phi_{t}$ anyway, so this does not add to the computational effort. To complete the set of equations, however, we need to express the corresponding boundary conditions satisfied by $\phi_{t}$ on the various boundaries.

We first note that taking the material derivative of the freeslip condition (2) yields the following relationship on $\Gamma_{c}(t)$ :

$\ddot{\mathbf{x}} \cdot \mathbf{n}+\dot{\mathbf{x}} \cdot \frac{\mathrm{d} \mathbf{n}}{\mathrm{d} t}=\left(\frac{\partial \nabla \phi}{\partial t}+\dot{\mathbf{x}} \cdot \nabla \nabla \phi\right) \cdot \mathbf{n}+\nabla \phi \cdot \frac{\mathrm{d} \mathbf{n}}{\mathrm{d} t}$

which gives, after reordering the terms, the following Neumann boundary condition on the body boundary:

$\frac{\partial \phi_{t}}{\partial n}(\mathbf{x}, t)=\frac{\mathrm{d} \mathbf{n}}{\mathrm{d} t} \cdot(\dot{\mathbf{x}}-\nabla \phi)+\ddot{\mathbf{x}} \cdot \mathbf{n}-\dot{\mathbf{x}} \cdot(\nabla \nabla \phi \cdot \mathbf{n})$

The assumption of rigid body motion yields, $\dot{\mathbf{x}}=\dot{\mathbf{x}}_{G}+$ $\dot{\theta}_{G} \times\left(\mathbf{x}-\mathbf{x}_{G}\right)$, where $\mathbf{x}_{G}$ is the location of the body center of mass and $\dot{\theta}_{G}$ is the body rotation vector. Here the advection velocity is the body velocity, but alternative numerical schemes could use the fluid velocity instead.

By applying the unsteady Bernoulli theorem, it is possible to derive the following partial differential equation, which must also be satisfied by the potential in $\Omega_{f}(t)$ :

$$
\frac{\partial \phi}{\partial t}+\frac{1}{2} \nabla \phi \cdot \nabla \phi+g z=-\frac{1}{\rho}\left(p-p_{a}\right)
$$

The Dirichlet boundary condition for $\phi_{t}$ on the free surface thus directly follows from the dynamic free surface boundary condition as:

$\frac{\partial \phi}{\partial t}=-\frac{1}{2} \nabla \phi \cdot \nabla \phi-g z$

Following Grilli and Subramanya (1996), in the 2D-HOBEM model, both the potential and its normal derivative (as well as their time derivative) are discretized by higher-order elements, here, both cubic. This allows computing gradients, 
such as in the previous equations, by taking the derivative of the shape functions within each boundary element. Specifically, we use so-called mixed cubic interpolation (MCI) elements, in which geometry is modeled by cubic splines and field variables are interpolated between each pair of nodes, using the mid-section of a four-node "sliding" isoparametric element.

Thus, assuming that the potential is known from the solution of the first problem and its gradient is numerically computed as detailed above, the second BIE problem for $\phi_{t}$ can be put in the same form as Eq. (3):

$$
\begin{aligned}
& \text { Find } \phi_{t} \text { such that: } \\
& \begin{array}{l}
c(\mathbf{x}, t) \phi_{t}(\mathbf{x}, t) \\
=\int_{\partial \Omega_{f}(t)}\left[\phi_{t, n}(\mathbf{y}, t) G(\mathbf{y}, \mathbf{x})-\phi_{t}(\mathbf{y}, t) \frac{\partial G}{\partial n}(\mathbf{y}, \mathbf{x})\right] \mathrm{d} S_{y} \\
\phi_{t}=-\frac{1}{2} \nabla \phi \cdot \nabla \phi-g z \text { on } \Gamma_{f}(t) \\
\phi_{t, n}=0 \text { on } \Gamma_{b} \text { and } \Gamma_{l} \\
\phi_{t, n}=\frac{\mathrm{d} \mathbf{n}}{\mathrm{d} t} \cdot(\dot{\mathbf{x}}-\nabla \phi)+\ddot{\mathbf{x}} \cdot \mathbf{n}-\dot{\mathbf{x}} \cdot(\nabla \nabla \phi \cdot \mathbf{n}) \text { on } \Gamma_{c}(t)
\end{array}
\end{aligned}
$$

where $\phi_{t, n}$ stands for $\frac{\partial^{2} \phi}{\partial n \partial t}$.

\subsection{Time updating}

The main flow chart of the 2D-BEM model solving wavestructure interactions is summarized in the Algorithm 1.

\subsubsection{Free surface time stepping}

For time updating the free surface and the potential, we propose to study the effect of two different schemes. The first one is the explicit second-order Taylor series, as used in the original 2D-FNPF code of Grilli and Svendsen (1990). This scheme has been widely validated for non-linear waves propagation purposes (Grilli et al. 2001) and is known to allow for larger time step than a first-order Euler scheme. In this explicit scheme, the new values of the potential $\phi$ and the position $\mathbf{x}$ at time $t_{i+1}$ may be expressed as follows:

$$
\left\{\begin{array}{l}
\phi^{i+1}\left(\mathbf{x}^{i+1}, t_{i+1}\right)=\phi\left(\mathbf{x}^{i}, t_{i}\right)+\frac{\mathrm{d} \phi}{\mathrm{d} t}\left(\mathbf{x}^{i}, t_{i}\right) \Delta t \\
+\frac{\mathrm{d}^{2} \phi}{\mathrm{d} t^{2}}\left(\mathbf{x}^{i}, t_{i}\right) \frac{\Delta t^{2}}{2} \\
\mathbf{x}^{i+1}=\mathbf{x}^{i}+\frac{\mathrm{d} \mathbf{x}}{\mathrm{d} t}\left(\mathbf{x}^{i}, t_{i}, \phi^{i}, \phi_{n}^{i}\right) \Delta t \\
+\frac{\mathrm{d}^{2} \mathbf{x}}{\mathrm{d} t^{2}}\left(\mathbf{x}^{i}, t_{i}, \phi^{i}, \phi_{n}^{i}\right) \frac{\Delta t^{2}}{2}
\end{array}\right.
$$

As mentioned by Dias and Bridges (2006), taking into acount the Hamiltonian structure of the equations may lead to a better performance of the algorithm for long time simulations. We thus propose to use a first-order scheme inspired by symplectic integrators:

$$
\left\{\begin{array}{l}
\phi^{i+1}\left(\mathbf{x}^{i+1}, t_{i+1}\right)=\phi\left(\mathbf{x}^{i}, t_{i}\right)+\frac{\mathrm{d} \phi}{\mathrm{d} t}\left(\mathbf{x}^{i}, t_{i}\right) \Delta t \\
\phi_{n}{ }^{i+1}\left(\mathbf{x}^{i+1}, t_{i+1}\right)=\phi_{n}\left(\mathbf{x}^{i}, t_{i}\right)+\frac{\mathrm{d} \phi_{n}}{\mathrm{~d} t}\left(\mathbf{x}^{i}, t_{i}\right) \Delta t \\
\mathbf{x}^{i+1}=\mathbf{x}^{i}+\frac{\mathrm{d} \mathbf{x}}{\mathrm{d} t}\left(\mathbf{x}^{i}, t_{i}, \phi^{i+1}, \phi_{n}^{i+1}\right) \Delta t
\end{array}\right.
$$

Using free surface conditions and denoting by $\phi_{s}$ the tangential derivative of the potential, we can further expand the first two expressions of Eq. (10) into:

$$
\left\{\begin{array}{l}
\phi^{i+1}=\phi^{i}+\Delta t\left(\phi_{t}^{i}+\phi_{s}^{i} \phi_{s}^{i}+\phi_{n}^{i} \phi_{n}^{i}\right) \\
\phi_{n}{ }^{i+1}=\phi_{n}^{i}+\Delta t\left(\phi_{t n}^{i}+\phi_{s n}^{i} \phi_{n}^{i}+\phi_{n}^{i}\left(\frac{1}{R^{i}} \phi_{n}^{i}-\phi_{s s}^{i}\right)\right)
\end{array}\right.
$$

where we made use of expressions given in Grilli and Subramanya (1993) for expressing the second-order normal derivative $\phi_{n n}$ with respect to the curvature $\frac{1}{R}$ and the secondorder tangential derivative $\phi_{s s}$. As can be seen in Eq. (10), this integrator uses new information on the potential and its normal derivative $\phi^{i+1}$ and $\phi_{n}^{i+1}$ at time $t_{i+1}$ to update the position to $\mathbf{x}^{i+1}$. However, the spatial derivatives are taken on the geometry at time $t_{i}$. Although we have no proof that the newly proposed scheme (10) possesses the properties of symplectic integrators (as defined by Lew et al. 2004), it will be referred to as symplectic-like scheme in the following. Similar schemes are known to have good energy-conserving properties on the long term, and have been widely used, e.g., in celestial mechanics and mechanical engineering (Lew et al. 2004).

\subsubsection{Body boundary time stepping}

We denote by $\mathbf{F}$ the force due to the total pressure acting on the body surface and by $\mathbf{X}$ the 6 components vector defined such that $X_{k}=x_{G k}$, for $k=1 \ldots 3$ and $X_{k}=\theta_{G k}$ for $k=4 \ldots 6$. The pressure has the following definition:

$p=-\rho\left(g x_{3}+\frac{1}{2} \nabla \phi \cdot \nabla \phi+\phi_{t}\right)$

For coupling the equation of motion to the fluid potential solver, we use a predictor-corrector scheme combined with a second-order Newmark scheme for the generalized velocity $\dot{\mathbf{X}}$ and the position $\mathbf{X}$ of the center of gravity which reads:

$$
\left\{\begin{array}{l}
\ddot{\mathbf{X}}_{i+1, k}=\mathbf{M}^{-1} \mathbf{F}_{i+1, k}^{*} \\
\dot{\mathbf{X}}_{i+1, k}=\dot{\mathbf{X}}_{i}+\Delta t\left((1-\beta) \ddot{\mathbf{X}}_{i}+\gamma \ddot{\mathbf{X}}_{i+1, k}\right) \\
\mathbf{X}_{i+1, k}=\mathbf{X}_{i}+\Delta t \dot{\mathbf{X}}_{i}+\frac{\Delta t^{2}}{2}\left(\left(\frac{1}{2}-\beta\right) \ddot{\mathbf{X}}_{i}+\gamma \ddot{\mathbf{X}}_{i+1, k}\right)
\end{array}\right.
$$

where $\mathbf{F}_{i+1, k}^{*}$ is the force correction in the sub-iteration number $k$ and $\mathbf{M}$ is the mass matrix of the body. The predictorcorrector scheme is initialized with a cubic extrapolation of the force exerted on the body at next time step $t_{i+1}$. The Newmark scheme has the advantage to better preserve the energy of conservative mechanical systems (see Kane 
1999) as compared to the classical fourh-order Runge-Kutta method. Besides, no sub-step is required for this scheme.

Between two sub-iterations, the boundary conditions for the Laplace problems are modified to take into account the new position and velocity of the solid:

$$
\left\{\begin{array}{l}
\phi_{n}^{i+1, k}=(\dot{\mathbf{x}} \cdot \mathbf{n})\left(\mathbf{X}^{i+1, k}, \dot{\mathbf{X}}^{i+1, k}\right) \\
\phi_{t n}^{i+1, k}=\left(\frac{\mathrm{d} \mathbf{n}}{\mathrm{d} t} \cdot(\dot{\mathbf{x}}-\nabla \phi)+\ddot{\mathbf{x}} \cdot \mathbf{n}-\dot{\mathbf{x}} \cdot(\nabla \nabla \phi \cdot \mathbf{n})\right) \\
\quad\left(\mathbf{X}^{i+1, k}, \dot{\mathbf{X}}^{i+1, k}, \ddot{\mathbf{X}}^{i+1, k}\right)
\end{array}\right.
$$

First, expressing the boundary condition on $\phi_{t n}$ in a local curvilinear coordinates system leads to the following formulation, as derived by Cointe (1989):

$$
\begin{gathered}
\phi_{t n}=\ddot{\mathbf{x}} \cdot \mathbf{n}+\left(\dot{\mathbf{x}} \cdot \mathbf{s}-\phi_{s}\right) \dot{\theta}_{G}-\left(\frac{1}{R} \phi_{s}+\phi_{s n}\right) \\
\dot{\mathbf{x}} \cdot \mathbf{s}+\left(\phi_{s s}-\frac{1}{R} \phi_{n}\right) \dot{\mathbf{x}} \cdot \mathbf{n}
\end{gathered}
$$

Based on these considerations, two numerical strategies may be adopted.

Implicit method. The first one follows the method used in Guerber et al. (2012) which is based on original works of van Daalen (1993) and Tanizawa (1995). The acceleration $\ddot{\mathbf{X}}$ is replaced in the Eq. (5) by the hydrodynamic force which makes it possible to express $\phi_{t}$ as a solution of another boundary integral problem whose support is restricted to the wetted body surface. This relationship takes the form:

$$
\phi_{t n}(\mathbf{x})+\int_{\partial \Omega_{b} \cap \Omega_{f}} K(\mathbf{x}, \mathbf{y}) \phi_{t}(\mathbf{y}) \mathrm{d} S_{y}=\gamma(\mathbf{x}), \quad \text { on } \partial \Omega_{b}(t)
$$

in which $K$ is a symmetrical kernel depending only on the rigid body geometry. For the expression of the function $\gamma$ and $K$, we refer to the work of Guerber et al. (2012). This extra integral equation may be incorporated in the influence matrix of the Laplace equation for more computational efficiency.

Direct method. From the expression derived by Cointe (1989), we also can use directly the acceleration given by the Eq. (13) for computing the new body boundary condition $\phi_{t n}$. In this case, no modification of the influence matrix is needed. A similar numerical scheme has been recently used in Yan and Ma (2007).

\subsubsection{Treatment of intersections between body and free surface}

In the BEM implementation, a double-node technique (Grilli and Svendsen 1990; Tanizawa 2000) is used to represent all intersection points between various parts of the boundary, including the solid body and the free surface. Such nodes have the same coordinates but are associated with two sets of unknowns, and in particular two normal gradients of the potential and its time derivative, with different normal directions. Let us denote by $x_{f}(t)=\left(\mathbf{x}_{i}^{f}(t)\right)_{i=1 \ldots N_{d}}$ the coordinates of $N_{d}$ nodes belonging to the intersection of the free surface and the body surface at time $t, \Gamma_{f}(t) \cap \Gamma_{c}(t)$. In the BEM model, these nodes are a priori associated with the outward normal vector on the free surface, denoted by $\mathbf{n}_{i}{ }^{f}\left(\mathbf{x}_{i}{ }^{f}(t), t\right)$. For each of these nodes, according to the double-node technique, we define a second set of nodes, $\mathbf{x}_{i}^{b}(t)$, having the same geometrical position but being associated with an inward normal vector on the body surface, $\mathbf{n}_{i}{ }^{b}\left(\mathbf{x}_{i}{ }^{b}(t), t\right)$. The nodes $\left(\mathbf{x}_{i}{ }^{f}(t)\right)_{i=1 \ldots N_{d}}$ are moving according to Eq. (9), while nodes $\left(\mathbf{x}_{i}^{b}(t)\right)_{i=1 \ldots N_{d}}$ are moving with the rigid body motion.

Due to the different trajectories of the free surface nodes and the body nodes at the intersection, it is necessary to redefine, at each time level, the double-node sets $x_{f}(t)$ and $x_{b}(t)$. In our developments, two distinct schemes have been tested for the determination of the new intersection points. In a first scheme, we project the new intersection $x_{f}(t+\Delta t)$ in the orthogonal direction on the new body surface $\Gamma_{c}(t+\Delta t)$. The drawback, however, is that the value of $\phi\left(x_{f}(t+\Delta t)\right)$ cannot be easily inferred. In the second scheme, we linearly extrapolate the position and the potential $\phi$ in the adjacent segment of the body on the surface $\Gamma_{f}(t+\Delta t)$. More precisely, if we denote by $\mathbf{x}_{i}^{f-1}$ (respectively, $\mathbf{x}_{i}^{b-1}$ ) the first neighbor node of $\mathbf{x}_{i}^{f}$ on the free surface (respectively, $\mathbf{x}_{i}^{b}$ on the body surface), we take the intersection between the straight line $\left(\mathbf{x}_{i}^{f-1}, \mathbf{x}_{i}^{f}\right)$ and the parametrization of the solid geometry. This intersection procedure has been only tested for elementary geometries such that the intersection between the discretized free surface and body surface can be computed analytically. For the case of a wall-sided cylinder (e.g., the rectangular barge), the new intersection point is thus given by the intersection of the straight line $\left(\mathbf{x}_{i}^{f-1}, \mathbf{x}_{i}^{f-1}\right)$ and the straight line $\left(\mathbf{x}_{i}^{b-1}, \mathbf{x}_{i}^{b}\right)$. For the case of the circular cylinder centered in $\mathbf{x}_{G}(t)$ and of radius $R$, we compute analytically the intersection of $\left(\mathbf{x}_{i}^{f-1}, \mathbf{x}_{i}^{f}\right)$ with the circle $\mathcal{C}\left(\mathbf{x}_{G}(t), R\right)$. Once the intersection has been computed, we extrapolate linearly the value of $\phi$ from the values $\left(\phi\left(\mathbf{x}_{i}^{f-1}\right), \phi\left(\mathbf{x}_{i}^{f}\right)\right)$. The drawback of the latter scheme is that it is quite difficult to generalize to a 3D geometry, while the first scheme has already been used in some 3D numerical wave tanks (Bai and Eatock Taylor 2009; Zhou et al. 2013). In the present applications, the second scheme was found to be more stable for solid bodies with curved boundaries, such as the semi-circular horizontal cylinder considered here, yielding a more regular time history for each component of the force. For this reason, results presented in the following are based on the extrapolation technique at the free surface. Note that also due to numerical errors, the velocity at the double nodes can be discontinuous. To avoid this discontinuity, Tanizawa's velocity compatibility condition (Tanizawa 1995) is applied to 
both the velocity and the acceleration vectors. This condition expresses the uniqueness of the velocity vector of the fluid particles $x_{f}(t)$, when expressed in the local basis attached to the solid geometry or in the local basis of the free surface geometry. This is similar to the condition devised by Grilli and Svendsen (1990) in the 2D-FNPF model.

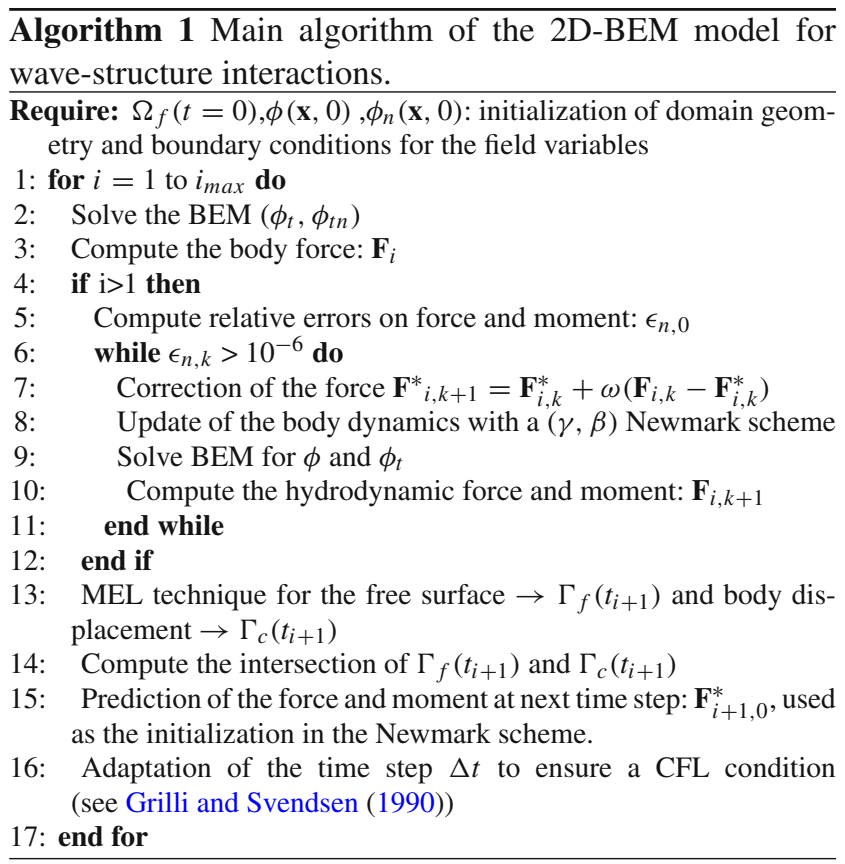

\section{Forced heaving motion of a circular horizontal cylinder}

In this section, we solve the case of the forced heaving motion of a horizontal circular cylinder (in a finite computational domain represented in Fig. 1), to check the accuracy and convergence of the numerical solution, including the hydrodynamic force, with respect to a reference solution. Since we eventually aim at predicting the motion of freely floating structures, the accuracy of the force calculation is of prime importance. For small to moderate amplitudes of motion, a reference solution can be obtained from linear or weakly non-linear theory, although it is difficult in a finite length numerical domain to simulate an infinite medium, such as assumed in the reference solutions.

More specifically, we consider the case of a semi-circular horizontal cylinder of radius $R$, heaving with a forced harmonic motion. The amplitude of the cylinder motion is first set to $0.01 R$, which generates a very small amplitude wave that should compare well with the prediction from linear theory. We then compare the vertical force obtained from a direct integration of the computed pressure on the instantaneous body wetted surface $\Gamma_{c}(t)$, to the first-order force predicted by linear theory. In our non-linear model, pressure is obtained from the unsteady Bernoulli equation (6).

For 2D problems, the hydrodynamic pressure force and moment applied to the floating body at time $t_{i}$ are given by:

$$
\begin{aligned}
F_{k}\left(t_{i}\right)= & \int_{\Gamma_{c}\left(t_{i}\right)}-\rho\left(g y_{3}+\phi_{t}(\mathbf{y})+\frac{1}{2} v^{2}(\mathbf{y})\right) \\
& \times n_{k}(\mathbf{y}) \mathrm{d} S_{y}, \quad k=1 \ldots 3 \\
F_{k}\left(t_{i}\right)= & \int_{\Gamma_{c}\left(t_{i}\right)}-\rho\left(g y_{3}+\phi_{t}(\mathbf{y})+\frac{1}{2} v^{2}(\mathbf{y})\right) \mathbf{e}_{k} \\
& \cdot\left\{\left(\mathbf{y}-\mathbf{x}_{G}\left(t_{i}\right)\right) \times \mathbf{n}(\mathbf{y})\right\} \mathrm{d} S_{y}, \quad k=4 \ldots 6
\end{aligned}
$$

where $v^{2}=\nabla \phi \cdot \nabla \phi$.

The last three components of the vector $\mathbf{F} \in \mathbb{R}^{6}$ are the three components of the moment in the basis $\left(\mathbf{e}_{1}, \mathbf{e}_{2}, \mathbf{e}_{3}\right)$. In a second stage, for larger amplitudes of motion up to $0.2 R$, we assess the model's ability to compute higher-order components of the force exerted on the cylinder.

In all cases, the conservation of the volume is computed as a function of the discretization. Finally, the $L^{2}$ norm error of various variables computed in the model is estimated by also computing the solution at internal nodes.

\subsection{Computational domain and simulation methodology}

We study the forced harmonic heaving motion of a rigid floating body, from a state of rest, which can be represented by the motion of its center of mass given by, $x_{G 3}(t)=$ $x_{G 3}(0)+A \sin (\omega t)$, with $A$ selected between $0.01 R$ and $0.2 R$. As noted before for wavemakers started from a state of rest (Grilli and Subramanya 1996), at $t=0$ there is a discrepancy between the initial (finite) velocity of the heaving
Fig. 1 Sketch of the 2D model computational domain at initial time, for the forced heaving motion of a horizontal semi-circular cylinder of radius $R$ in water depth $d$. Numbers indicate the codes associated with each type of boundary, with boundary conditions marked on the figure

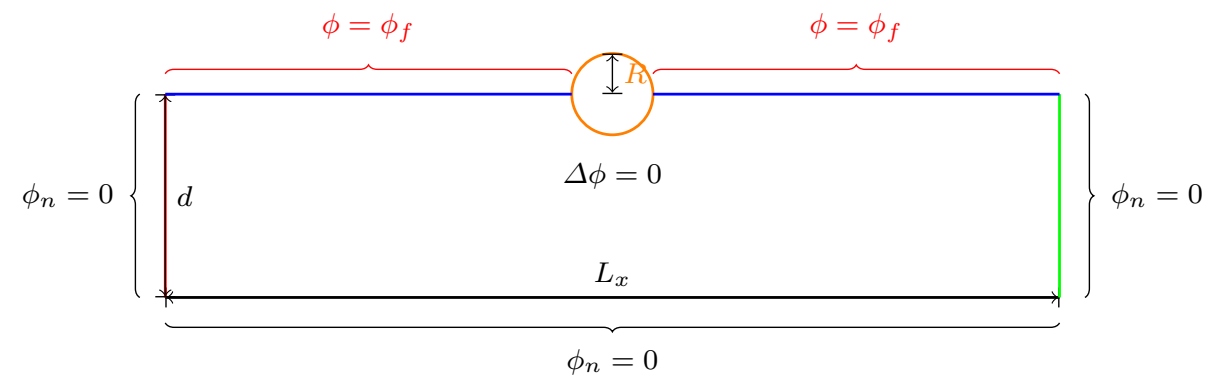


body and that of the free surface nodes. If large, this incompatibility can trigger high wave number instabilities. To limit this problem, the body is gradually set in motion according to the following equation:

$x_{G 3}(t)=x_{G 3}(0)+D(t) A \sin (\omega t), \quad t \leq t_{\text {ramp }}$

where $D(t)$ is a ramp function defined by:

$D(t)=\frac{\tanh \left\{C\left(\frac{t}{t_{\text {ramp }}}-1\right)\right\}+\tanh C}{\cosh \left\{C\left(\frac{t}{t_{\text {ramp }}}-1\right)\right\}(1+\tanh C)}$

where the constant $C$ is set to $C=2.3025$ and the ramp-up time $t_{\text {ramp }}=5 T$, with $T=2 \pi / \omega$ the period of the heaving motion. A typical geometry of the computational domain is shown in Fig. 1. The rigid floating body is located in the middle of a 2D wavetank of length $L_{x}$ and depth $d$. At the tank extremities, in the absence of an exact radiation condition, we use a full reflective wall by setting the Neumann condition $\phi_{n}=0$ (left and right boundaries in Fig. 1). A uniform space step between nodes $\Delta x$ is used over the whole boundary of the computational domain. As in earlier work (Grilli and Subramanya 1996; Guerber et al. 2012), time step $\Delta t$ is automatically selected in the model, based on an optimal mesh Courant number, $\mathcal{C}_{0}=c \Delta t / \Delta x=0.45$, where $c$ is the dominant wave celerity.

To eliminate reflection from sidewalls, which would eventually affect the force computation, following Bai and Eatock Taylor (2006), we specify absorbing beaches over a length of $2 \lambda$ from each wall, where $\lambda$ is the characteristic wavelength of the waves radiating from the heaving body. In the absorbing beaches $(\mathrm{AB})$, an "absorbing" free surface pressure is added to the dynamic free surface boundary condition (6), leading to:

$\frac{\mathrm{d} \phi}{\mathrm{d} t}=-g z+\frac{1}{2} \nabla \phi \cdot \nabla \phi-v(\mathbf{x}) \phi$

Similarly, a dissipative term is also introduced in the projection of the kinematic free surface boundary consition on the vertical axis, and is defined like so:

$\frac{\mathrm{d} z}{\mathrm{~d} t}=\phi_{z}-v(\mathbf{x}) z$

where the damping coefficient has the following definition:

$v(\mathbf{x})= \begin{cases}\omega\left(\frac{x_{1}-x_{\mathrm{AB}}}{l_{\mathrm{AB}}}\right)^{2} & x_{1} \geq x_{\mathrm{AB}} \\ 0 & x_{1}<x_{\mathrm{AB}}\end{cases}$

where $x_{\mathrm{AB}}$ is the coordinate on the horizontal axis at the entrance of the beach and $l_{\mathrm{AB}}$ denotes the length of the beach. The performance of the absorption has been measured for $N_{f}=30$ nodes per wavelength, by computing the reflection coefficient $K$ defined as:

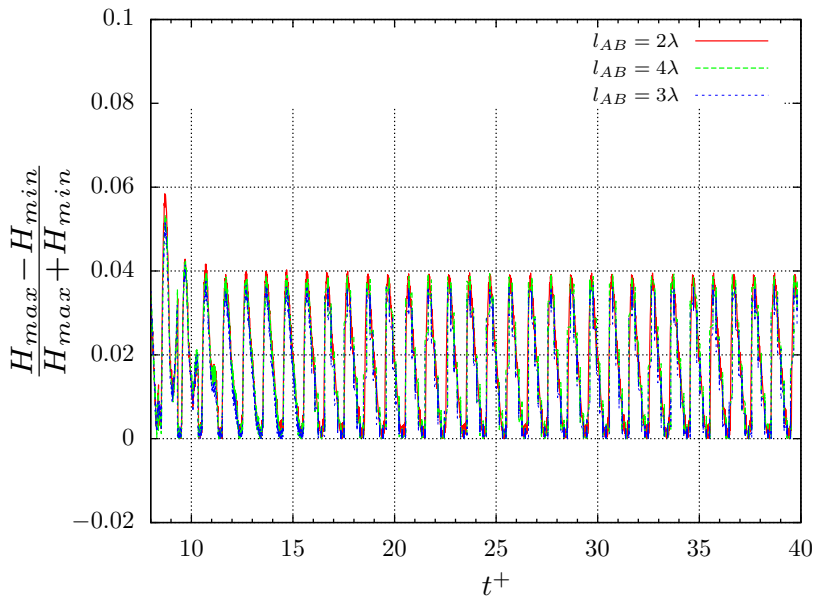

Fig. 2 Forced oscillation of a circular cylinder: evaluation of the reflection coefficient $K$ for $\mathrm{A}=0.01 R$ according to the size of the beach $l_{\mathrm{AB}}$

$K=\frac{H_{\max }-H_{\min }}{H_{\max }+H_{\min }}$

where $H_{\max }$ (respectively, $H_{\min }$ ) denotes the maximum (respectively, the minimum) wave height in an area between the body and the entrance of the absorbing beach. The efficiency of this absorbing system is evaluated with the small amplitude $A / R=0.01$, which gives rise to a regular wave pattern. In this case, the coefficient $K$ is computed for three different lengths of the $\mathrm{AB}$ beach and is represented in Fig. 2. The efficiency of the AB beach appears not to be sensitive to its length, as each configuration leads to a coefficient $K$ close to $4 \%$. Furthermore, it is observed that the present system provides a constant damping for long-term simulations, when monochromatic waves are to be simulated. Based on these tests, the numerical wave tank is equipped on both sides by a beach of length $l_{\mathrm{AB}}=2 \lambda$ for forced motions computations.

We study the convergence of the numerical solution towards the reference solution, for a given angular frequency of motion $\omega$, such that $\omega^{2} R / g=1.0$. In deep water, this non-dimensional frequency is equal to $k R$, i.e., it is proportional to the ratio between the width of the body at the free surface and the deep water wavelength $\lambda_{0}=2 \pi / k$ (with $k$ the wavenumber), of the radiated waves caused by the body heaving motion. The computational domain length is selected such that $L_{x} \gg \lambda_{0}$, typically $L_{x}=10 \lambda_{0}$. In general, the linear dispersion relationship reads, $\omega^{2}=\frac{2 \pi}{\lambda} g \tanh \frac{2 \pi}{\lambda} d$. In the applications, the NWT is set to $d=\lambda_{0}$, which more than satisfies the usual deep water wave condition. To compare our results with the results of Kent (2005), which are presented in non-dimensional form, the acceleration of gravity is set to $g=1 \mathrm{~m}^{2} / \mathrm{s}$, the fluid density to $\rho=1 \mathrm{~kg} / \mathrm{m}^{3}$, and the cylinder radius to $R=1 \mathrm{~m}$; in this case, $\lambda_{0}=2 \pi \approx 6.28 \mathrm{~m}$. Results are plotted with respect to the non-dimensional time $t^{+}=t / T$. To assess the long-term behavior of the numerical 
scheme and verify results for volume conservation, simulations are carried out up to $t_{\max }=15 T\left(t_{\max }^{+}=15\right)$.

Because the absorbing beaches cover a length $2 \lambda$ at each extremity of the NWT, wave-structure interactions can be studied over the central part of the domain of length $6 \lambda$. To assess the effects of the absorbing beaches, simulations will be repeated in some cases without the absorbing pressure. It will be seen that wave reflection has a very significant impact on the conservation of global quantities such as the total fluid energy, denoted $E_{f}(t)$, and the total fluid volume, denoted $V\left(\Omega_{f}(t)\right)$.

We solve the forced heaving problem for seven different meshes, whose characteristics are detailed in Table 1. Each mesh was selected such that, when the body and the free surface are at rest, the space step is uniform on the boundary. However, given the temporal character of our simulations, the grid size is kept constant only within each part of the boundary. As indicated before, after each iteration, regridding is applied to the free surface boundary to prevent BEM nodes from getting too close from each other on the free surface, which is known to trigger high wave-number instabilities (see Dommermuth and Yue 1987; Grilli and Svendsen 1990). To this end, the length of each part of the boundary, represented by piecewise cubic splines (respectively, linear functions) for the free surface nodes (respectively, for the body nodes) is first computed and the nodes are redistributed uniformly.

\subsection{Numerical error on volume conservation}

For each simulation, with parameters listed in Table 1, the conservation of the computational domain volume is assessed as a function of the discretization (parameter $N_{f}$ ) with and without absorbing beaches. Without absorption in the beaches, the total fluid energy in the NWT will keep increasing, owing to the external forcing of the rigid body motion, which acts as a wavemaker. This will cause wave steepness to keep increasing, as reflected waves combine with incident waves, eventually leading to strong non-linearity and overturning, which will gradually affect the numerical accuracy. Note that the entrance of the beach is located at $x_{\mathrm{AB}}=2 \lambda_{0}=4 \pi$.

Figure 3 shows the time evolution of the relative error in total volume conservation during 15 periods of heaving motion, for three different discretizations, two amplitudes of motion, for weakly non-linear $A / R=0.01$ and strongly non-linear $A / R=0.2$ waves, and with or without absorbing beaches. For small amplitude motion, the first-order symplectic-like scheme achieves an error of nearly $\mathcal{O}\left(10^{-7}\right)$ which is 2 order of magnitude better than the explicit second-order Taylor series. Moreover, with the symplecticlike scheme, this error is not increasing after 15 periods. For the second-order explicit Taylor series, the use of an absorbing beach seems to reduce the relative error on the volume, which does not occur when using the symplectic-like scheme. This suggests, that the later scheme has better properties for conserving the volume. For each time stepping, the simulation of the larger amplitude motion $A / R=0.2$ gives a conservation of the volume 2 order of magnitude worse than with the small amplitude $A / R=0.01$.

This error analysis indicates that, for large wave steepness or cylinder amplitude of motion, the second-order Taylor time stepping with MCI BEM elements on the free surface, does not conserve volume well enough and may thus become inaccurate, as time increases. Although errors on volume increase with time, in all cases, except at the very beginning of simulations, this increase is monotonous, likely due to the use of the regridding method on the free surface. For large amplitude of motion, as energy is fed into the wavetank and wave steepness increases, the initial discretization based on a flat free surface may become too coarse as compared to the initial stages of computations, which may result in increased numerical errors.

\subsection{Numerical error on hydrodynamic force calculation}

We now analyze the relative numerical error on hydrodynamic force calculation with respect to a known reference solution (index ref), as a function of the domain discretization and other parameters of the problem. For each discretization $N_{f}$ listed in Table 1, we define the relative error on the force

Table 1 Forced heaving motion of a circular cylinder: discretization used in the model for the convergence study

\begin{tabular}{llllllll}
\hline$\lambda / \Delta x=N_{f}$ & 10 & 20 & 30 & 40 & 50 & 60 & 80 \\
\hline$\Delta x=2 \pi / N_{f}$ & 0.63 & 0.32 & 0.21 & 0.16 & 0.12 & 0.10 \\
$N_{c}$ & 10 & 10 & 15 & 20 & 25 & 30 & 40 \\
$N_{\text {el }}$ & 224 & 444 & 669 & 894 & 1,119 & 1,344 \\
$N_{\text {dof }}$ & 231 & 451 & 676 & 901 & 1,126 & 1,351 \\
\hline
\end{tabular}

For the selected parameters, $\lambda=\lambda_{0}=2 \pi, N_{\text {dof }}$ denotes the total number of BEM nodes (or degrees of freedom) and $N_{\mathrm{el}}$ the total number of boundary elements. $N_{f}$ is the number of BEM nodes per dominant wavelength and $N_{c}$ is the number of BEM nodes discretizing the half-cylinder boundary 
Fig. 3 Forced oscillation of a circular cylinder: time evolution of the relative error of volume $\frac{V(t)-V_{0}}{V_{0}}$ in $\log _{10}$ scale as a function of the discretization $N_{f}$ (varying from 20 to 60 from left to right) as a function of: (i) the amplitude of motion $A$ (from the top $A / R=0.01$ to the bottom $A / R=0.2$ ); (ii) with or without absorbing beaches; and (iii) the time-stepping (scheme A second-order explicit time stepping; scheme $B$ first-order symplectic-like time stepping)
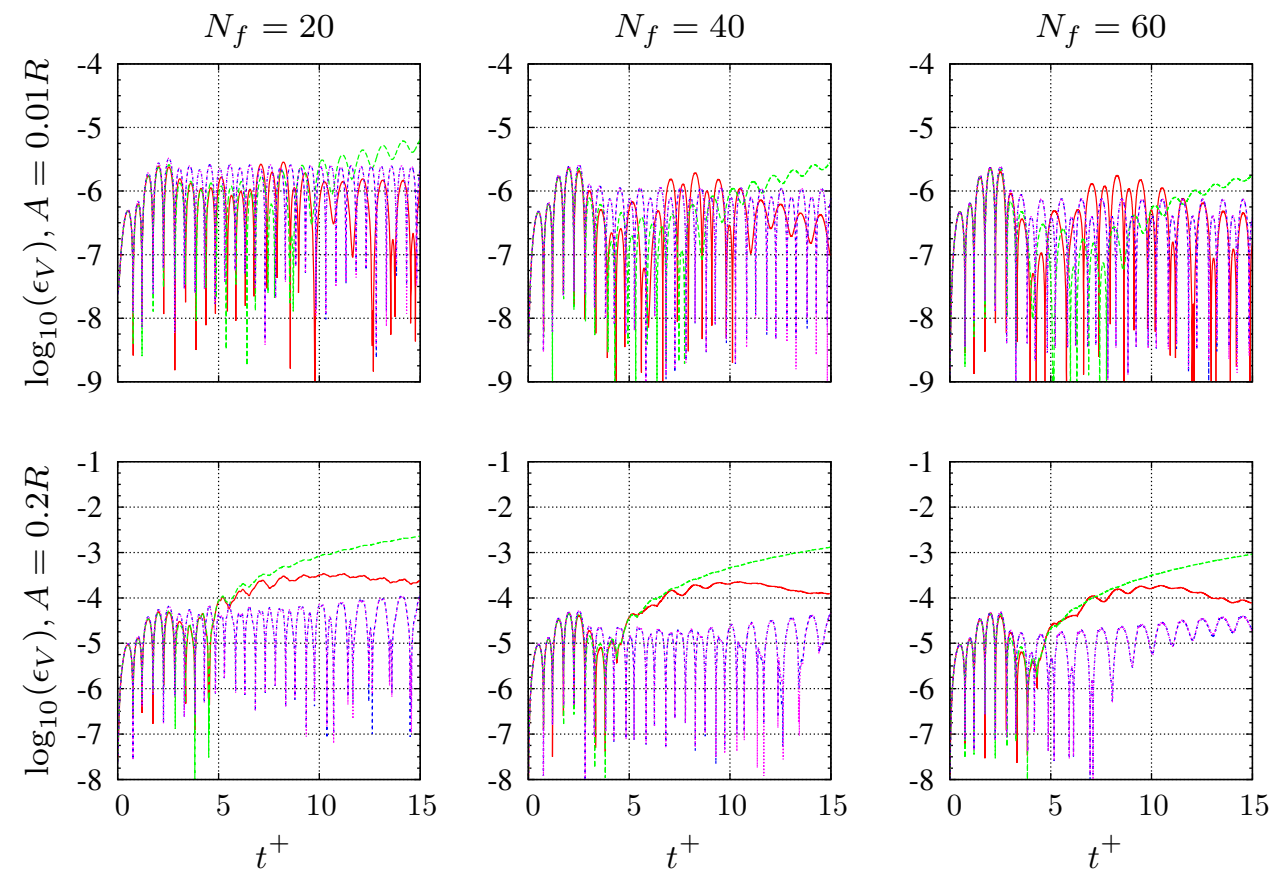

scheme A AB beach

scheme A no AB

scheme $\mathrm{B} A \mathrm{AB}$ beach

scheme $\mathrm{B}$ no $\mathrm{AB}$

coefficients satisfy the relationship:

$F_{3}(t)=-a_{33}(\omega) \ddot{x}_{G 3}(t)-b_{33}(\omega) \dot{x}_{G 3}(t)$

$\epsilon_{N_{f}}\left(t_{n}\right)=\sqrt{\frac{\left\{f_{N_{f}}\left(t_{n}\right)-f_{\text {ref }}\left(t_{n}\right)\right\}^{2}}{\max _{n}\left\{f_{\text {ref }}\left(t_{n}\right)^{2}\right\}}}$

where $t_{n}$ is the discrete time corresponding to the $n$-th time step.

An indicator of the global error on the hydrodynamic force computation, for a simulation lasting from time $t_{i}$ to time $t_{f}$, is finally defined by taking the average of the $\epsilon_{N_{f}}\left(t_{k}\right)$ for $t_{k}=t_{i}$ to $t_{f}$ as:

$\epsilon_{N_{f}}\left(t_{i}, t_{f}\right)=\frac{1}{\left(n_{f}-n_{i}\right)} \sum_{n=n_{i}}^{n_{f}} \epsilon_{N_{f}}\left(t_{n}\right)$

\subsubsection{Small amplitude motion}

Using absorbing beaches and a fine enough grid, we find that the hydrodynamic force becomes quasi-periodic from $t / T=5$ to 10 (as can be seen in the test case of a rectangular barge in sway motion in Fig. 8). The stable force signal between $t / T=5$ and 10 is Fourier transformed into the frequency domain, from which one can compute the standard hydrodynamic coefficients of the body heaving motion, namely the added mass $a_{33}$ and damping $b_{33}$ coefficients. Let us recall that, to the limit of linear wave theory, these which gives the amplitude of the force in phase with the body motion as, $F_{3}^{\text {in }}=a_{33}(\omega) A \omega^{2}$ and that of the force out of phase with this motion as, $F_{3}^{\text {out }}=-b_{33}(\omega) A \omega$. During the time interval under consideration, $a_{33}$ and $b_{33}$ are computed and made non-dimensional by the following transformation: $a_{33}^{*} \leftarrow F_{3}^{\text {in }} /\left(\rho \frac{\pi}{2} \omega^{2} R^{2}\right)$ and $b_{33}^{*} \leftarrow F_{3}^{\text {out }} /\left(\rho \frac{\pi}{2} \omega^{2} R^{2}\right)$. The hydrodynamic coefficients and the Fourier analysis in the following are computed with the help of the software Scilab. Table 2 shows the values of these coefficients computed in our model for various discretization $N_{f}$, compared to other firstorder values reported in the literature, by Kent (2005), Frank (1967), Porter (1960). A fairly good agreement is observed between all these first-order quantities and our results for the two finest discretizations $\left(N_{f}=60\right.$ and 80$)$.

Figure 4 illustrates the global convergence of the force computation with the discretization, by computing the global error $\epsilon_{N_{f}}\left(t_{i}, t_{f}\right)$, defined by Eqs. (24) and (25), between $t_{i}=0.16 T$ and $t_{f}=19.7 T$ (with $T=2 \pi$ based on selected parameters), as a function of $N_{f}=20$ to 60 , using as a reference value the force $f_{N_{f}}$ computed for $N_{f}=80$. We see that, for a small amplitude motion, $A / R=0.01$, the error decreases as the discretization is refined, confirming the convergence of our numerical scheme. 
Table 2 Forced heaving motion of a semi-circular cylinder of radius $R$ in deep water: non-dimensional hydrodynamic coefficients $\left(a_{33}^{*}, b_{33}^{*}\right)$ computed with our model using $N_{f}=20-80$ nodes per dominant wavelength $\left(A / R=0.01, \omega^{2} R / g=1.0\right)$, compared to reference values from the literature

\begin{tabular}{ll}
\hline$N_{f}=20$ & $(0.57353,0.44863)$ \\
$N_{f}=40$ & $(0.59644,0.42155)$ \\
$N_{f}=60$ & $(0.60063,0.41302)$ \\
$N_{f}=80$ & $(0.60172,0.40929)$ \\
Porter $(1960)$ & $(0.58,0.41)$ \\
Frank $(1967)$ & $(0.62,0.40)$ \\
Kent $(2005)$ & $(0.582,0.410)$ \\
\hline
\end{tabular}

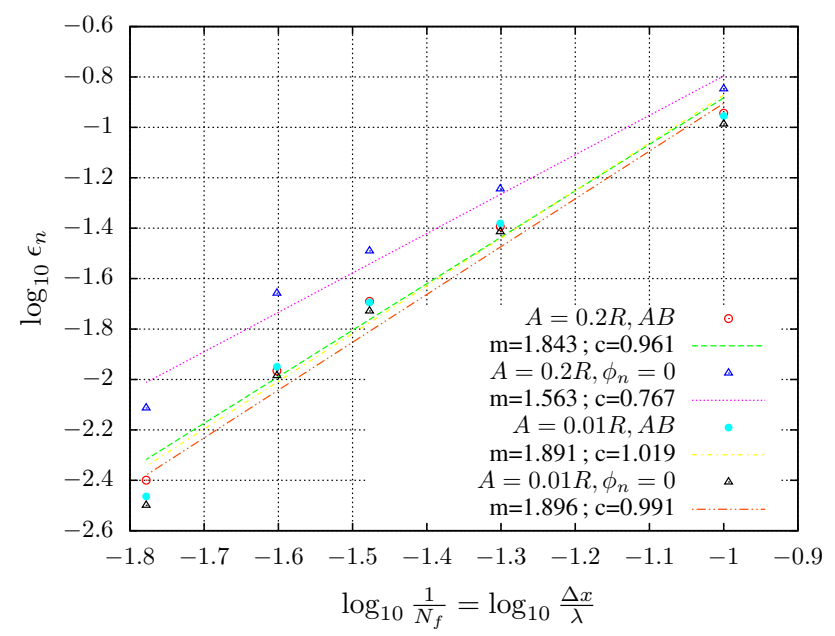

Fig. 4 Forced heaving motion of a semi-circular cylinder of radius $R$ in deep water: global error on the force computation as a function of the discretization $N_{f}=20$ to 60 . Results are computed over the time interval $t_{i}=0.16 T$ to $t_{f}=19.7 T$ and the force reference value $f_{N_{f}}$ is that computed in the finest discretization with $N_{f}=80$. The coefficients $m$ and $c$ are determined by computing a least mean square trend line of equation $y=m x+c$ for each set of points

The rate of convergence, in the least mean square sense, may be evaluated with an absorbing beach, as 1.891 for $A / R$ $=0.01$ and 1.843 for $A / R=0.2$. Without absorbing beach, it takes the values 1.896 for $A / R=0.01$ and 1.563 for $A / R=0.2$. These values are quite satisfactory given the fact that we used linear elements for meshing the body geometry and the force depends on the solution on the body nodes only. These computations highlight also the fact that the use of an absorbing beach may improve the accuracy of the force calculation at high amplitudes.

\subsubsection{Large amplitude motion}

We now similarly study the accuracy and convergence of the computation of the hydrodynamic force for a large amplitude of motion of the heaving cylinder. This problem was experimentally studied by Yamashita (1977), who tested a
Table 3 Forced heaving motion of a semi-circular cylinder of radius $R$ in deep water: Fourier coefficients of the hydrodynamic force

\begin{tabular}{lllll}
\hline & $N_{f}=20$ & $N_{f}=40$ & $N_{f}=60$ & $N_{f}=80$ \\
\hline$c_{0}$ & 0.05437 & 0.06375 & 0.06534 & 0.06629 \\
$c_{1}$ & 0.23299 & 0.23225 & 0.23159 & 0.23075 \\
$c_{2}$ & 0.01979 & 0.02234 & 0.02248 & 0.02232 \\
$c_{3}$ & 0.00031 & 0.00055 & 0.00034 & 0.00044 \\
\hline
\end{tabular}

Results are computed over the time interval $t^{+}=11.14$ to 16.08 as a function of the discretization $N_{f}$, for $A / R=0.2$ and $\omega^{2} R / g=1$

few 2D horizontal cylinders with different cross-sections, including elliptical cylinders and bow stern sections, and compared experimental results to the weakly non-linear solution of Lee (1968). Based on these results, in a following section, we will assess the accuracy of the present FNPF model in computing actual measured forces. Consistent with earlier results presented here, we restrict ourselves to a semicircular floating cylinder oscillating with a large amplitude motion $A / R=0.2$.

As before, we assess the model convergence in this more strongly non-linear case by comparing values computed for a coarser discretization to those for the finest discretization with $N_{f}=80$. Besides the convergence of the total hydrodynamic force for a sufficiently fine mesh, it is also expected that each coefficient of the Fourier series of the signal should converge to the approximate value predicted by weakly nonlinear theories. Denoting by $c_{n}$ the Fourier coefficient of order $n$ and using the notations of Yamashita (1977), the Fourier decomposition of the vertical component of the total hydrodynamic force $F_{3}(t)$ reads:

$F_{3}(t)=c_{0}-2 \sum_{k \geq 1}\left|c_{k}\right| \sin \left(k \omega t+\delta_{k}\right)$

where $\delta_{k}$ is the phase associated with the $k$-th component.

The rate of convergence of the force for large amplitude motion is seen in Fig. 4 to be 1.843 with an absorbing beach and 1.563 without beach. As already discussed above for small amplitude motion, it is seen in Fig. 4 that, without absorbing beaches, the errors are much larger; this results from the large energy trapping in the wavetank that yields increasingly non-linear and under-resolved waves. Regarding the Fourier coefficients of the force $F_{3}(t)$, Table 3 lists $c_{n}$ values computed up to third-order for various discretizations, using Eq. (27), for $t / T=5$ and 10. As before, convergence is nearly achieved for $N_{f}=60$, except for the third-order force coefficient, which is associated with strongly non-linear effects.

\subsection{Convergence of results for internal points}

In this section, for the same application to the forced heaving motion of a semi-circular cylinder of radius $R$ in deep water, 
Fig. 5 Forced heaving motion of a semi-circular cylinder of radius $R$ in deep water: instantaneous $L^{2}$ error norm over time $t \in[0,10 T]$, in the computation of internal fields $\phi$, $\phi_{t}$ and $p$, as a function of the discretization $N_{f}=20$ (red), 40 (green) and 60 (blue), with amplitude $A / R$, as compared to the reference values computed with the finest discretization, $N_{f}=80$
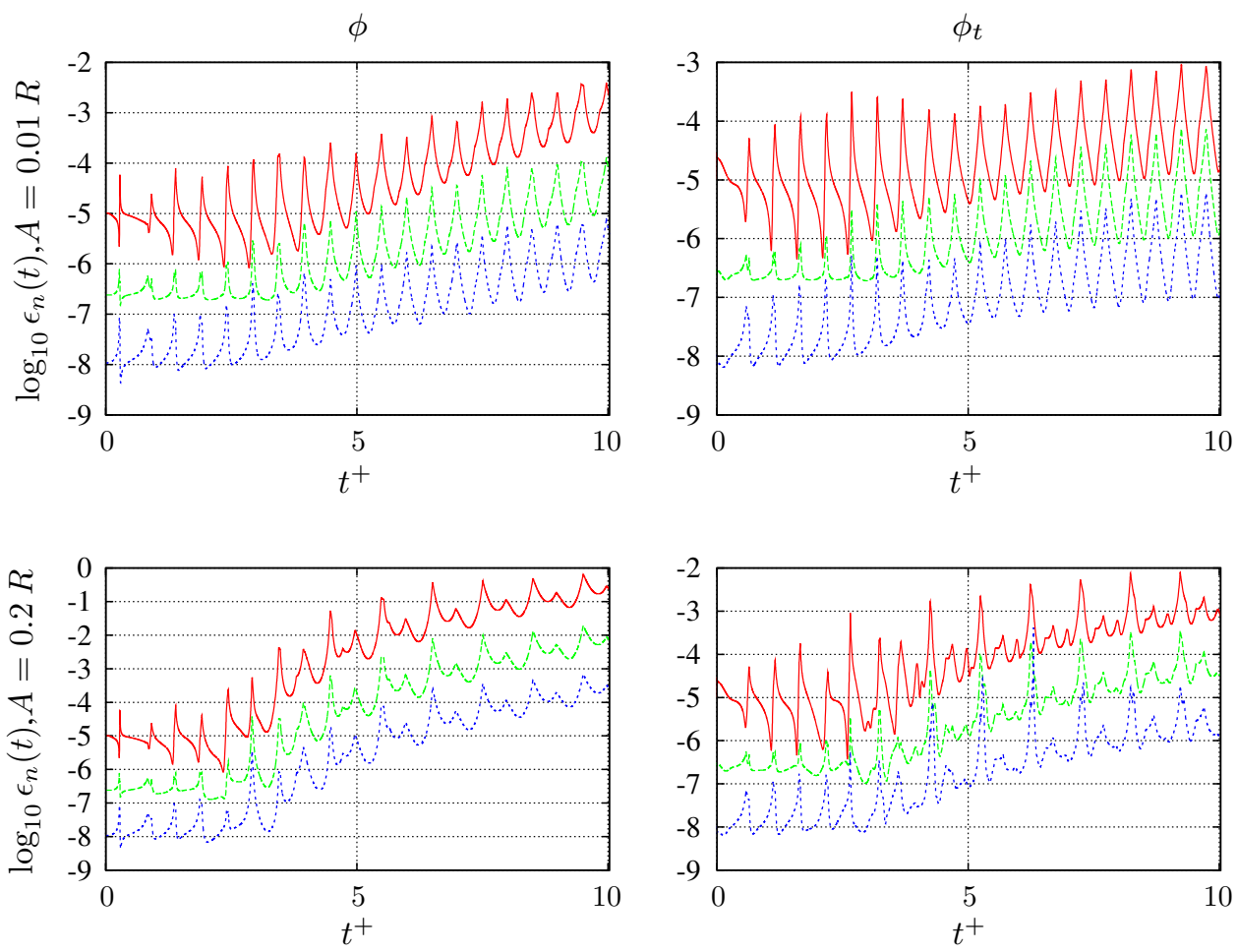

we assess the instantaneous and global $L^{2}$ convergence of various model results computed at internal points (i.e., within the fluid domain), when refining the discretization $\left(N_{f}\right)$. We will solve both small and large amplitude motion cases, with $A / R=0.01$ and 0.2 , respectively, using the same fixed time step $\Delta t=0.005 \mathrm{~s}$.

The internal points under consideration are located in such a way that they remain inside the domain during the whole simulation, to avoid an ambiguous comparison for elements located at the free surface. In the model, the solution at internal points satisfies the same BIE as for boundary points, except that $c(\mathbf{x}, t)=1$, and the boundary fields $\phi$ and $\phi_{t}$ (and their normal derivative) are assumed to be known at time $t_{i}$. Hence the computation of the solution at internal points is explicit. Thus, assuming a pre-defined grid of internal points of coordinates $\mathbf{x}_{\text {gages }}$ within the domain, the velocity potential at internal points is given by $\left(i=1, \ldots, N_{\text {gages }}\right)$ :

$$
\begin{array}{r}
\phi\left(\mathbf{x}_{\text {gages }}, t_{i}\right)=\int_{\partial \Omega_{f}\left(t_{i}\right)}\left[\phi_{n}\left(\mathbf{y}_{k}, t_{i}\right) G\left(\mathbf{x}_{\text {gages }}, \mathbf{y}_{k}\right)\right. \\
\left.-\phi\left(\mathbf{y}_{k}, t_{i}\right) \frac{\partial G}{\partial n}\left(\mathbf{x}_{\text {gages }}, \mathbf{y}_{k}\right)\right] \mathrm{d} S_{y}
\end{array}
$$

on the discretized geometry $\Omega_{f}\left(t_{i}\right)$ at time $t_{i}$. Other variables, such as the internal velocity can also be explicitly obtained by applying the gradient operator to the Green's function within the integral kernel, from which the pressure can be computed using Bernoulli equation.
Here, to simplify and reduce the post-processing steps, a simple internal grid is considered. Assuming a first geometry of the wavetank with, $L_{x}=62.3 \mathrm{~m}$ and $d=6.3 \mathrm{~m}$, "numerical" gages are specified at a series of discrete $x$-locations, $x_{i}=1$ to $x_{f}=60 \mathrm{~m}$, with an uniform step $\Delta x_{\text {gages }}=3 \mathrm{~m}$ and, in the vertical direction, from $y_{i}=-2$ to $y_{f}=-6 \mathrm{~m}$, by steps $\Delta y_{\text {gages }}=1 \mathrm{~m}$. For this particular case, $N_{\text {gages }}=100$. As indicated, besides $\phi$ and $\phi_{t}$, which reflect the accuracy of the BEM solution, we will also assess the convergence of the variable $p$, which is numerically derived from the BEM solution, by way of the time derivative of the BIE (28) and its spatial gradient, which provide $\phi_{t}$ and the velocity, respectively. In this manner, the accuracy of the time stepping is also verified.

We compute $\phi, \phi_{t}$ and $p$ at internal points, for three boundary discretizations with $N_{f}=20,40$ and 60, and calculate the instantaneous $L^{2}$ error norm for each of these variables, compared to the results obtained with the finest discretization $\left(N_{f}=80\right)$. Results for both amplitudes of motion are displayed in Fig. 5. When these errors are time averaged for $N_{\text {iter }}$ time steps, as (e.g., for the potential $\phi$ ):

$\epsilon_{N_{f}}=\frac{1}{N_{\text {iter }}} \sum_{i=1}^{N_{\text {iter }}} \sqrt{\frac{1}{N_{\text {gages }}} \sum_{k=1}^{N_{\text {gages }}} \frac{\left(\phi_{N_{f}}\left(x_{k}, t_{i}\right)-\phi_{\text {ref }}\left(x_{k}, t_{i}\right)\right)^{2}}{\max _{x_{k}} \phi_{r e f}{ }^{2}}}$

it is possible to evaluate a global $L^{2}$ error norm associated with a given simulation. Note, the square root in Eq. (29) 
Fig. 6 Forced heaving motion of a semi-circular cylinder of radius $R$ in deep water: same case as Fig. 5, global $L^{2}$ error norm for $\phi, \phi_{t}$ and $p$, obtained by averaging instantaneous errors for $t=0$ to $10 T$. The coefficients $m$ and $c$ are determined by computing a least mean square trend line of equation $y=m x+c$ for each set of points

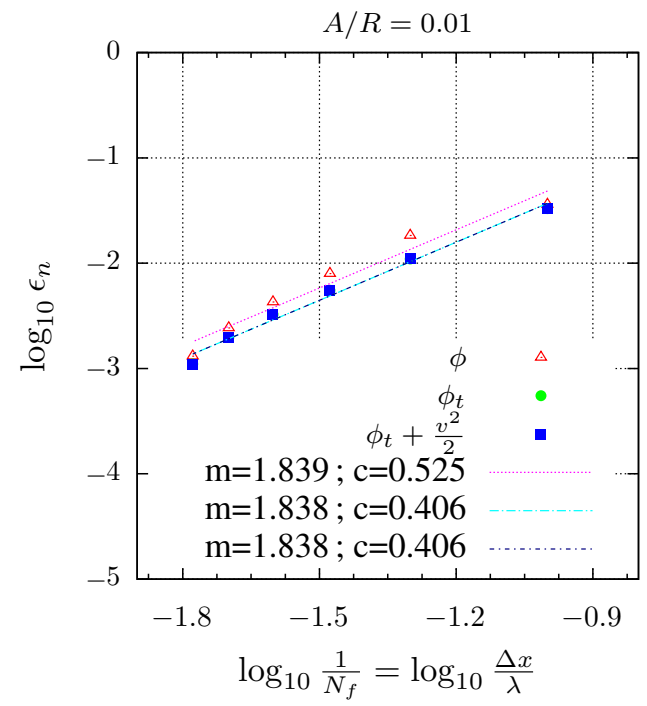

represents $\epsilon_{N_{f}}(t)$. These global convergence indicators are represented in Fig. 6, from which it is apparent that the order of the spatial convergence, for a fixed time step, is not constant over the set of tested discretizations (i.e., not a constant power of $N_{f}$ ). Nevertheless, if we compute the root mean square line for the set $\left(\log \frac{1}{N_{f}}, \log \epsilon_{N_{f}}\right)$, it is seen that the order of convergence of these simulations is close to 1.83 for the small amplitude $A / R=0.01$ and close to 1.88 for the larger amplitude $A / R=0.2$. These computations were done using the second-order Taylor explicit time stepping.

\subsection{Comparison to the experiments of Yamashita}

We now propose to repeat the same numerical simulation for a set of discrete frequencies to get a comparison with the experiment conducted by Yamashita (1977). Each simulation is carried out using the methodology presented before for the convergence study. The signal is analyzed on a temporal window included in the time interval $t^{+} \in[5,10]$. The computed coefficients are then made non-dimensional as done by Yamashita by taking:

$c_{2}{ }^{*}=\frac{2 c_{2}}{2 \rho g R^{2} \epsilon^{2}}$

$c_{3}{ }^{*}=\frac{2 c_{3}}{2 \rho g R^{2} \epsilon^{3}}$

with $\epsilon=\frac{A}{R}=0.2$. The added mass $a_{33}$ and the damping coefficient $b_{33}$ are also calculated and made non-dimensional by $a_{33}^{*}=\frac{a_{33}}{\rho \pi \frac{R^{2}}{2}}$ and $b_{33}^{*}=\frac{b_{33} \sqrt{\frac{R}{g}}}{\rho \pi \frac{R^{2}}{2}}$. On the upper part of Fig. 7, the hydrodynamic coefficients previously defined are compared to the theoretical predictions of Tasai and Koterayama (1976) and to the experimental values measured by Yamashita (1977). A rather good agreement is observed for these two quantities at least in the middle range of fre- quencies. For low frequency, the experimental results and the theory of Tasai are not in agreement, which can be explained by the inviscid assumption of the model. The damping coefficient measure appears to be the most affected by viscous effects, which seems reasonable. The high-order force coefficients $c_{2}$ and $c_{3}$ are presented on the lower panels of Fig. 7. An excellent agreement with the low-order theory of Lee (1968) is observed for $c_{2}$ in the low to middle frequency range. For $\frac{\omega^{2}}{g} R \geq 1$, the value of $c_{2}$ computed by the present FNPF model becomes greater than the prediction of the weakly nonlinear theory. For the third-order coefficient $c_{3}$, it appears that our calculations underpredict the results from the low-order theory. By contrast, we observe a slightly better agreement of our results with the experimental values at low and high frequencies. This could result from an insufficient mesh resolution for computing high-order harmonics as it is seen in Table 3 that the third-order Fourier coefficient does not reach a clear limit even for the very fine discretization with 80 nodes per wavelength. Moreover, it is also seen in Table 3, that the dimensional coefficient $c_{3}$ has a very small absolute value, which makes it more difficult to converge on $c_{3}$ than on $c_{1}$ for given values of the numerical parameters. The satisfactory agreement for first-order and second-order quantities seems to suggest that, for the ratio $A / R=0.2$, the approximate theories are valid, and confirm the convergence of the non-linear model towards these theories.

\section{Forced swaying motion of a rectangular floating cylinder}

Here, we verify the convergence of numerical results to the predictions of linear theory for a rectangular barge in forced swaying motion. Rather than performing another exhaustive convergence study for a single frequency, as we did for the 
Fig. 7 Forced heaving motion of a semi-circular cylinder of radius $R=B / 2$ in deep water: first- to third-order Fourier non-dimensional coefficients of the hydrodynamic force, for a large motion amplitude $A / R=0.2$. Results are computed over the time interval $t^{+} \in[5,10]$ as a function of the excitation frequency $\omega^{2} B /(2 g)$ : FNPF computations (red circles); experiments of Yamashita (1977) (blue squares); Tasai and Koterayama (1976) and Lee (1968) theories (green lines)
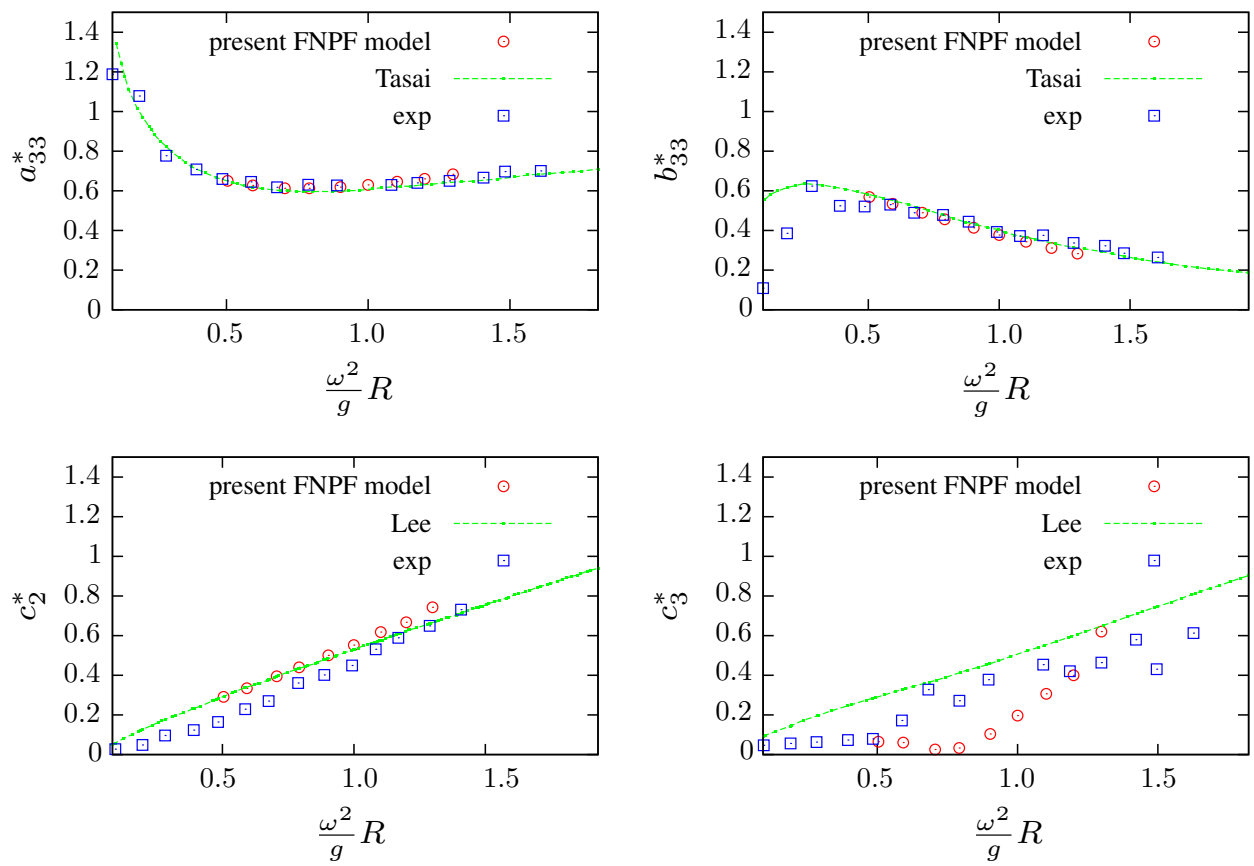

semi-circular cylinder, we focus instead on the accuracy of the force calculation over a wide range of frequencies, as we did in Sect. 3.5. Again, the discretization is specified to be uniform over the computational domain boundary. The discretization over the body surface is thus adjusted to be the same as on the free surface. A discretization based on $N_{f}=$ 80 nodes per wavelength is used in all cases, which has been shown in earlier applications to ensure a good convergence of numerical results. The barge draft is set to $a=1 \mathrm{~m}$ and its width to $B=2 a=2 \mathrm{~m}$. The amplitude $A$ of the swaying motion is set to a small value corresponding to $\frac{A}{a}=0.01$, which will make the comparison with linear theory relevant. The force hydrodynamic coefficients are calculated over the interval $t^{+}=t / T \in[5.84,10.84]$ (see in Fig. 8).

The hydrodynamic coefficients in sway $a_{22}$ and $b_{22}$ as well as the cross-coupling coefficients between sway (translation along the $O x$ axis) and pitch (rotation around the $O y$ axis) $a_{24}$ and $b_{24}$ are defined so as to verify:

$$
\begin{aligned}
& F_{2}(t)=-a_{22}(\omega) \ddot{x}_{G 2}(t)-b_{22}(\omega) \dot{x}_{G 2}(t) \\
& F_{4}(t)=-a_{24}(\omega) \ddot{x}_{G 2}(t)-b_{24}(\omega) \dot{x}_{G 2}(t)
\end{aligned}
$$

where $F_{2}$ is the horizontal component of the force along the $O x$ axis and $F_{4}$ is the transverse moment in the $y$ direction.

Figure 9 shows the added mass $a_{22}$ and damping $b_{22}$ hydrodynamic coefficients in sway, as well as those corresponding to the cross-coupling between sway and pitch, $a_{24}$ and $b_{24}\left(a_{24}\right.$ is the added inertia in pitch due to the forced motion in sway), computed as a function of angular frequency. In the figure, our results are compared to Vugts' experiments and computations based on linear theory. The numerical results of Vugts have been digitized from figures

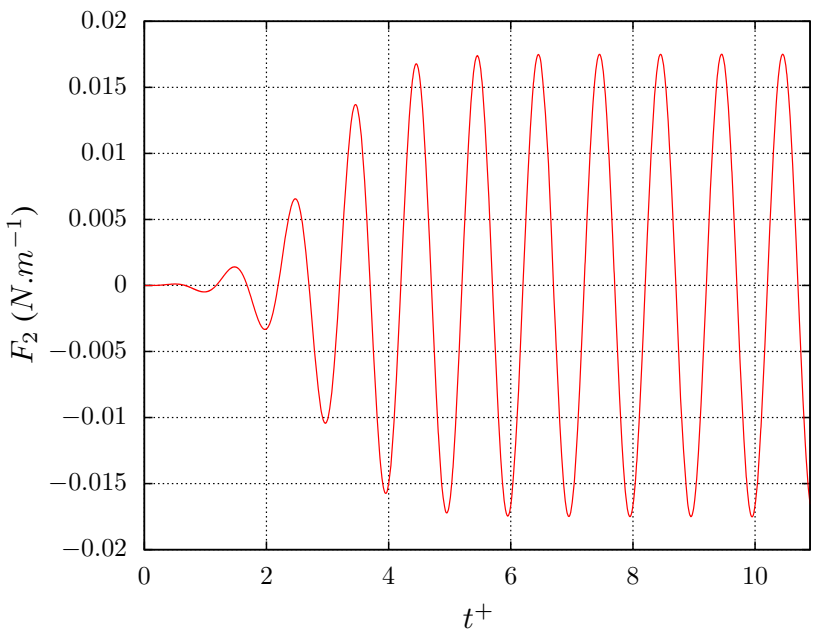

Fig. 8 Sway motion of a rectangular barge: horizontal force at frequency $\omega=0.84 \mathrm{~s}^{-1}$ represented on the time interval $t^{+}=t / T \in$ $[0,10.84]$

in the paper of Andersen and Wuzhou (1985). Vugts experimental results have been digitized thanks to the curves presented in the review of Wehausen (1971). The parameters used to make the coefficients non-dimensional are selected as in Andersen and Wuzhou (1985) (see definitions in the figure). The angular frequency is made non-dimensional as $\omega^{*}=\omega \sqrt{a / g}$.

We observe a fairly good agreement of our results with linear theory over the whole frequency range, both for the components of the force, both in and transverse to the barge direction of oscillation. This calculation confirms the ability of the model to accurately compute very fine scale phenom- 
Fig. 9 Rectangular barge of width $B$ and draft $a$ in swaying motion: added mass and damping coefficients as a function of the non-dimensional angular frequency. Comparison between numerical wave tank (red circles), to both linear theory (green line) and experimental (blue squares) results of Vugts (1968)
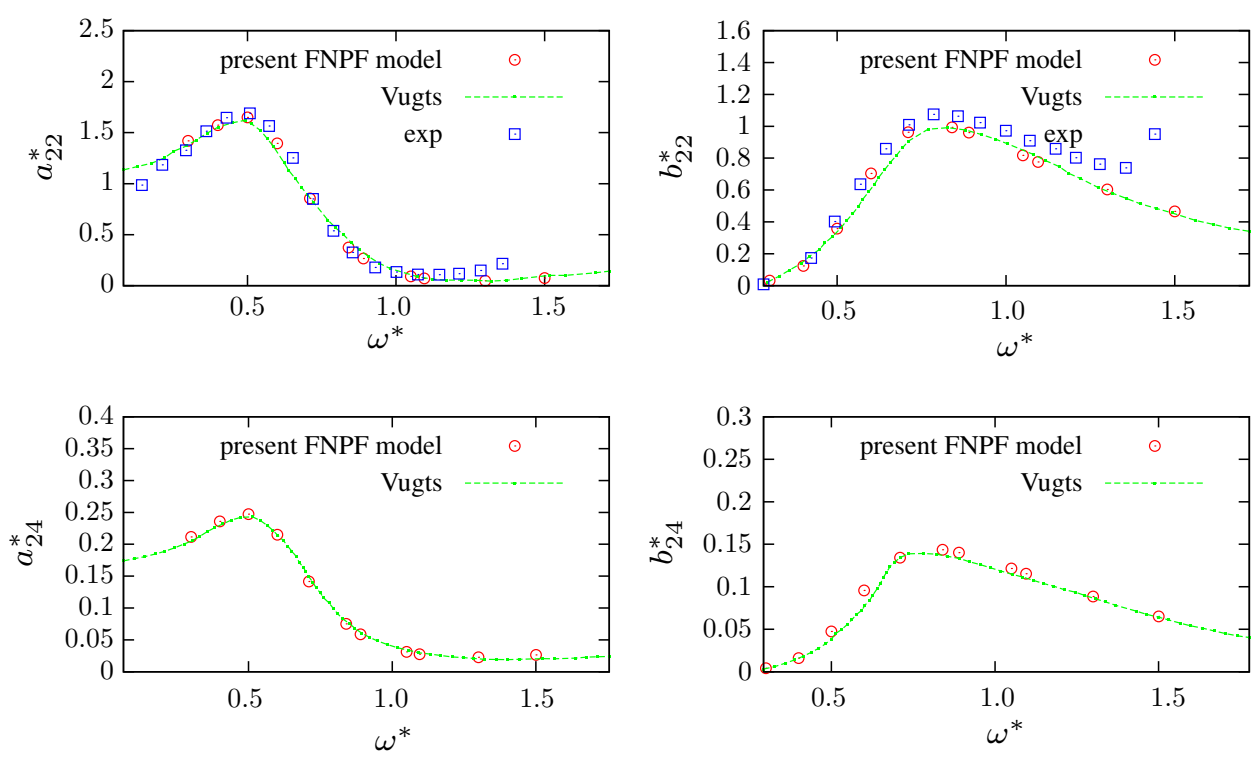

ena, such as the cross-coupling components of the hydrodynamic force. However, we note that these nearly linear calculations (as well as results from the linear theory) do not match all of Vugts' experimental data. In particular, we observe that linear results overestimate the added mass $a_{22}$ at low frequency, for $\omega^{*} \leq 0.3$, and underpredict it at high frequencies, for $\omega^{*} \geq 1.2$. In between, the agreement with experiments is quite good. This discrepancy is even more significant for the damping coefficient $b_{22}$, which is underestimated by linear theory mainly for higher frequencies. The discrepancy with the experimental values at low frequencies may be explained by the fact that, for long waves with respect to the body size, viscous effects become gradually more important (Fig. 9).

\section{Freely floating circular cylinder}

Having confirmed in earlier applications the accuracy and convergence of this 2D-FNPF model for forced heaving or swaying motions of various horizontal cylinders, we now assess the accuracy of the proposed wave-structure coupling algorithm, for the free oscillations of a floating circular cylinder released from a non-equilibrium position. In the linear limit, Maskell and Ursell (1970) derived a semianalytical solution of the vertical displacement of the center of mass, $x_{G 3}(t)$ for: (i) a zero initial velocity condition $\left(x_{G 3}(0) \neq 0, \dot{x}_{G 3}(0)=0\right)$; or (ii) a zero displacement initial condition $\left(x_{G 3}(0)=0, \dot{x}_{G 3}(0) \neq 0\right)$.

Below, we first detail the geometry of the computational domain and its discretization. Then, we check the convergence with respect to the discretization of results computed at internal points. Finally, we study in detail the cylinder oscillations as a function of time, for two different numerical
Table 4 Freely floating circular cylinder case: discretizations used in the convergence study for internal points computations. Number of nodes: on the free surface $\left(N_{F}\right)$; on the cylinder $\left(N_{c}\right)$; and total $\left(N_{\text {dof }}\right)$

\begin{tabular}{llllll}
\hline & Mesh 1 & Mesh 2 & Mesh 3 & Mesh 4 & Mesh 5 \\
\hline$\Delta x(\mathrm{~m})$ & 0.131 & 0.098 & 0.049 & 0.033 & 0.024 \\
$N_{F}$ & 150 & 200 & 250 & 300 & 400 \\
$N_{c}$ & 25 & 25 & 25 & 25 & 25 \\
$N_{\text {dof }}$ & 364 & 476 & 926 & 1,376 & 1,826 \\
\hline
\end{tabular}

schemes, and assess the overall accuracy of computations by checking conservation of mass and total energy (body plus fluid), and the sensitivity of errors on mass and energy conservation to the spatial discretization.

\subsection{Computational domain and discretization}

We consider a 2D rectangular domain of length $L_{x}=20 \mathrm{~m}$ and depth $d=5 \mathrm{~m}$, and study the free oscillations of a semicircular cylinder of radius $R=0.25 \mathrm{~m}$, which is released from a non-equilibrium position $x_{G 3}(0) \neq 0(R / 3, R / 4$ and $R / 8)$. Time is made non-dimensional as, $\tau=t \sqrt{g / R}$ and the cylinder motion evolution in terms of $x_{G 3}(t)$ is studied for $\tau \in[0,20]$. Parameters for the 5 initial discretizations used in the convergence study are given in Table 4. In each case, the solution is computed at internal points vertically spaced $\Delta y=1 \mathrm{~m}$, specified along $N_{\text {gages }}=153$ vertical numerical gages distributed over the fluid domain, horizontally spaced $\Delta x=0.5 \mathrm{~m}$. Over these points, a $L^{2}$ error norm is computed for the result difference with respect to the finest (fifth) mesh, which has $N_{F}=400$ nodes on the free surface. 
Fig. 10 Freely floating cylinder of radius $R$ : convergence of results at internal points $(5$ meshes; Table 4), expressed by the $L^{2}$ error norm of the solution for the 4 coarser grids as compared to that in the fifth finest grid. The cylinder center of mass is initially set to $x_{G 3}(0)$. These results were obtained with the second-order explicit Taylor time stepping

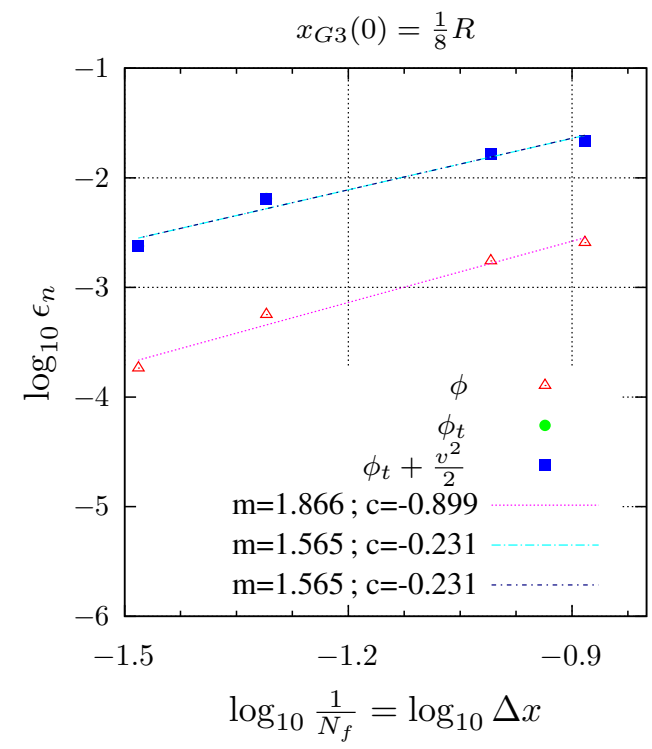

\subsection{Convergence of results at internal points}

The $L^{2}$ error norm of the variables $\phi, \phi_{t}$ and $p$ relative to results in the finest grid is displayed in Fig. 10. For the first initial displacement $x_{G 3}(0)=R / 8$, we also tested the aforementioned direct scheme, which consists in replacing directly in Eq. (5) the velocity and the acceleration of the rigid body given by the Newmark scheme. This is somewhat similar to the Iterative "Semi Implicit Time Integration Method for Floating Bodies (ISITIMFB)" recently presented in Yan and Ma (2007). Unless otherwise stated, this scheme will be referred to as the direct method. For this scheme, the relaxation parameter in the correction step of the Newmark scheme was set to $\omega=0.7$ as this was observed to minimize the number of sub-iterations required to achieve convergence of the hydrodynamic force. Note that this relaxation parameter is set to $\omega=1$ for the implicit method. Additionally, the other parameters of the Newmark scheme are set in each case to $\gamma=0.5$ and $\beta=0.25$.

In Fig. 10, the $L^{2}$ error norms related to the variables $\phi$, $\phi_{t}$ and $p$ are represented for different discretizations. Note that the $L^{2}$ error of $\phi_{t}$ and $p$ is superimposed. The root mean square straight lines are computed for each set of points. It can be observed that the order of convergence and the accuracy on the calculation of the variable $\phi$ are better than that for $\phi_{t}$ and $\phi_{t}+\frac{1}{2} v^{2}$. The rate of convergence is approximately 1.87 for $\phi$ while it reaches the value 1.56 for the other variables. For the amplitude $x_{G 3}(0)=R / 4$, the rate of convergence is slightly greater than for the amplitude $x_{G 3}(0)=R / 8$. This time, we observe that the level of the accuracy on the variable $\phi_{t}$ is worse than the one on $\phi$. This effect could be due to the sub-iterations used for the convergence of the force calculation. It is worth noting, as in the case of forced motion,

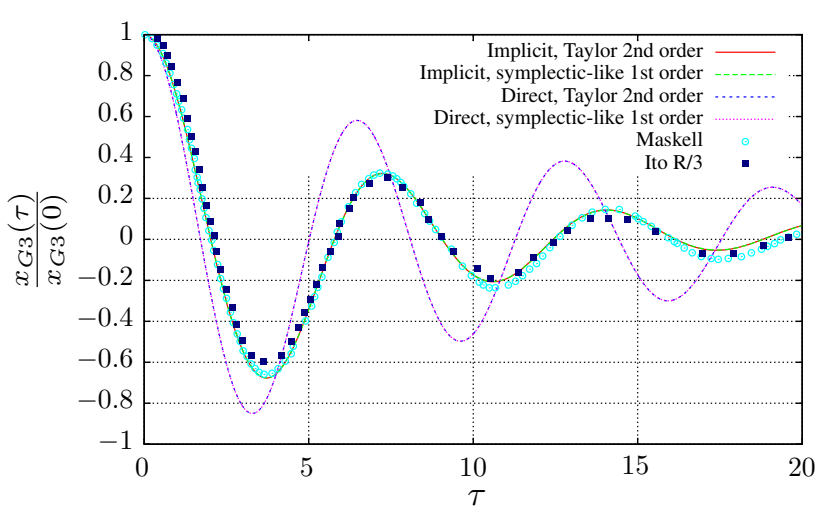

Fig. 11 Freely floating cylinder of radius $R$ in deep water: time evolution of the elevation of the cylinder, initially located at $x_{G 3}(0)=R / 8$, as a function of numerical scheme compared to reference values in linear theory for a small initial displacement of the structure with $N_{c}=50$ nodes on the body surface. The green dotted line and the red line are quasi-superimposed. The blue dotted line and the pink dotted line are quasi-superimposed

that the rate of convergence is slightly better for large motion than for small motion amplitude.

\subsection{Time evolution of the cylinder elevation as a function of numerical scheme}

In Fig. 11, we compare the computed time evolution of the cylinder elevation, for $x_{G 3}(0)=R / 8$, using the direct method or the implicit one, to the semi-analytical method of Maskell and Ursell (1970); and (ii) to the experimental values of Ito (1977). The experiments of Ito were performed with various initial displacements: 1 in, 1.5 in and 2 in and for a diameter of the cylinder equal to $2 R=6$ in (in the foot- 


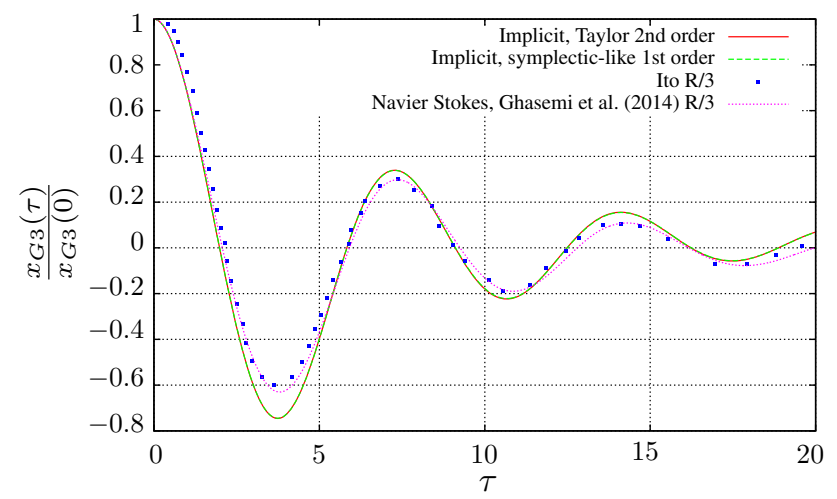

Fig. 12 Freely floating cylinder of radius $R$ in deep water: time evolution of the elevation of the cylinder, initially located at $x_{G 3}(0)=R / 3$, as a function of numerical scheme compared to reference values in linear theory for a strong initial displacement of the structure with $N_{c}=50$ nodes on the body surface

pound system). It was found that the first two amplitudes gave almost the same free decay curve (which is the one displayed in Fig. 11). Results were computed for the finest mesh (5) with $N_{F}=400$. In Fig. 12, the computed time evolution of the cylinder elevation, for $x_{G 3}(0)=R / 3$ is represented and compared to the Navier-Stokes computations of Ghasemi et al. (2014).

It can be concluded that results obtained using the implicit method are in much better agreement with the reference solutions than results using the direct method for the smallest amplitude $x_{G 3}(0)=R / 8$. Moreover, we note that the agreement with the linear predictions of Maskell and Ursell appears to be satisfactory only for the smallest amplitude $x_{G 3}(0)=R / 8$. For the other amplitude $R / 3$, the vertical displacement predicted by the present method significantly deviates from the prediction of linear theory after the first minimum of the displacement for $\tau \approx 4$.0. In Fig. 12, it is seen that the model of Ghasemi et al. (2014) based on a twofluid flow Navier-Stokes method gives a better prediction of the vertical displacement compared to experimental work of Ito (1977). This unfortunate behavior of the present FNPF model could be explained by the absence of viscous effects.

In terms of computational efficiency, running the implicit method with the discretization $N_{F}=400$ requires a constant number of 3 sub-iteration in the Newmark scheme, while the direct method requires 5-6 sub-iterations to achieve convergence. Based on these tests, the direct method strategy does not appear as a viable alternative to the implicit method. We will thus use the implicit method in our 3D model.

\subsection{Verification of mass and energy conservation}

For the earlier results, we also computed the time evolution of total energy of the fluid-body system and total fluid volume. The total energy of the fluid-body system can be split into the fluid energy $E_{f}(t)$, consisting of kinetic and potential energy, and the body mechanical energy $E_{b}(t)$. Energy conservation in these inviscid simulations imposes that total energy should be constant at any time step. More precisely, in the model, the fluid energy is expressed as:

$E_{f}(t)=\frac{1}{2} \rho g \int_{\Gamma_{f}(t)}(z+d)^{2} \mathrm{~d} s+\frac{1}{2} \rho \int_{\Gamma_{f}(t)} \phi \phi_{n} \mathrm{~d} s$

and the solid body mechanical energy reads:

$E_{b}(t)=m g\left(x_{G 3}+d\right)+\frac{1}{2} \dot{\mathbf{x}}_{G}^{T} \mathbf{M} \dot{\mathbf{x}}_{G}$

where $m\left(\mathrm{~kg} \mathrm{~m}^{-1}\right)$ is the body mass per unit length and the inertia matrix $\mathbf{M}$ is diagonal with, $M_{11}=M_{33}=m$ and $M_{22}=I_{G}$, where $I_{G}$ denotes the moment of inertia around the $y$ axis passing through the body center of mass. The conservation of energy states that:

$\Delta E_{f}(t)=-\Delta E_{b}(t)$

We also know that the variation of the mechanical energy of the solid is equal to the work done by the force acting on it, that is $\Delta E_{b}(t)=W=\int_{0}^{t} \mathbf{F} \cdot \dot{\mathbf{x}}_{G} \mathrm{~d} t$. Conservation of total energy is checked in the model by computing the relative error, $\Delta\left(E_{f}(t)+E_{b}(t)\right) / E_{b}(0)$ as a function of time, with the initial fluid energy being referred to zero and the denominator being the initial mechanical energy given the solid body by its initial vertical displacement; this is done in the top panels of Fig. 13, for two different discretizations and two initial amplitudes. In the middle panels of the figure, we computed $\left(\Delta E_{b}-W\right) / E_{b}(0)$, which enables to check the second conservation principle. Finally the bottom panels of the figure show the evolution of the relative error on fluid volume conservation, $\left(V\left(\Omega_{f}(t)\right)-V_{0}\right) / V_{0}$. The latter error stays very small in all cases, although it increases with time and is larger, the larger the initial cylinder displacement. This confirms the accuracy of both Laplace equation solver and time stepping schemes used in the present 2D-FNPF model.

It is worth noting that, for the two discretizations tested here with $N_{F}=100$ and 400, the conservation of energy is satisfied within the same degree of accuracy. Note that the discretization of the body was kept constant for each discretization of the free surface so that only the influence of this latter parameter was studied. This shows that if we use a fixed time step, refining the mesh at the free surface does not improve the accuracy of the energy conservation when the discretization of the body is kept constant. This reflects the fact that numerical errors on energy conservation can only decrease with grid refinement if the Courant number is held constant. When using an adaptive time step and the same discretizations as before, we obtain the results shown in Fig. 14. It is seen that the the accuracy on the volume ratio $\left(V\left(\Omega_{f}(t)\right)-V_{0}\right) / V_{0}$ and on the energy ratio $\left(\Delta E_{b}-\right.$ $W) / E_{b}(0)$ is significantly improved when changing both the space and time discretizations according to a CFL condition, 
Fig. 13 Freely floating cylinder of radius $R$ : time behavior of 3 energy and volume ratios for the minimal and maximal discretizations and for two initial release amplitudes $x_{G 3}(0)=R / 8$ and $x_{G 3}(0)=R / 4$ with the same fixed time step. These results were obtained with the second-order explicit Taylor time stepping
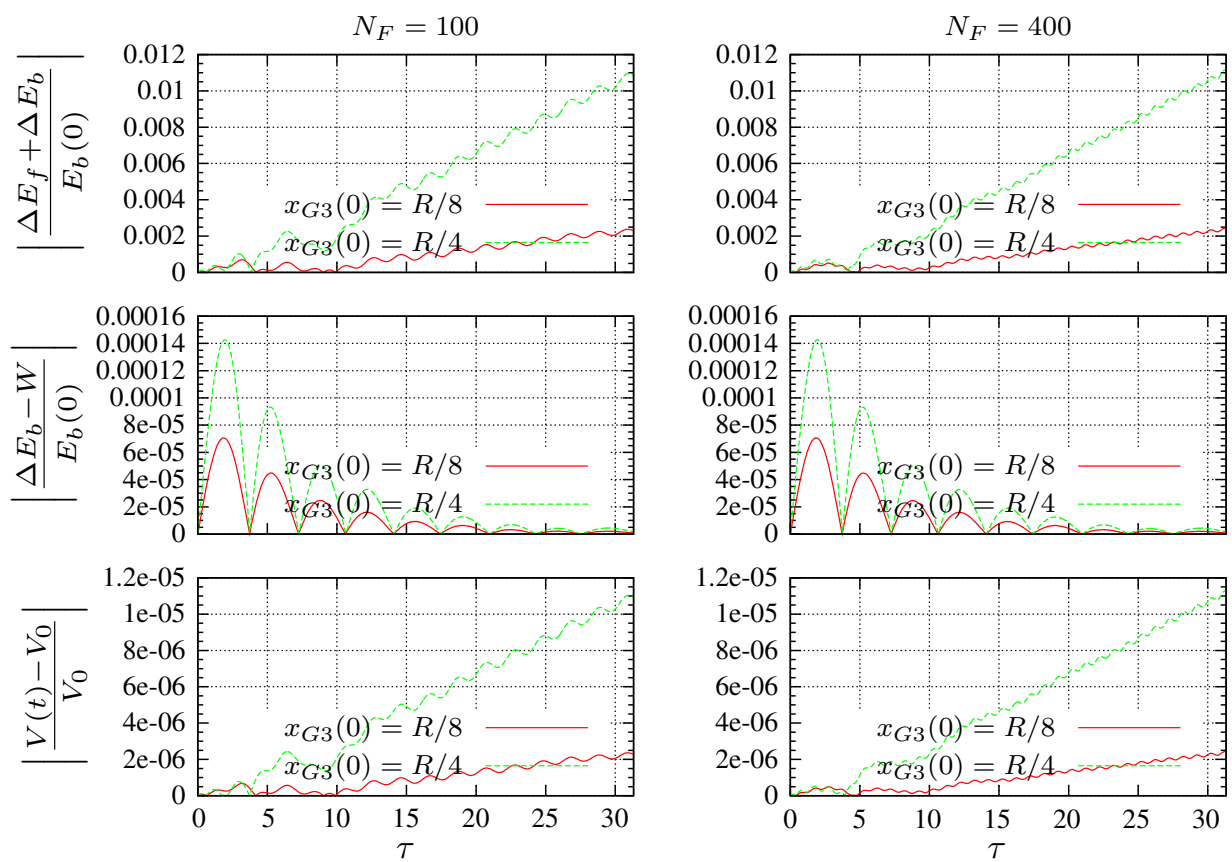

Fig. 14 Freely floating cylinder of radius $R$ : same case as Fig. 13 with an adaptive time-step procedure. These results were obtained with the second-order explicit Taylor time stepping
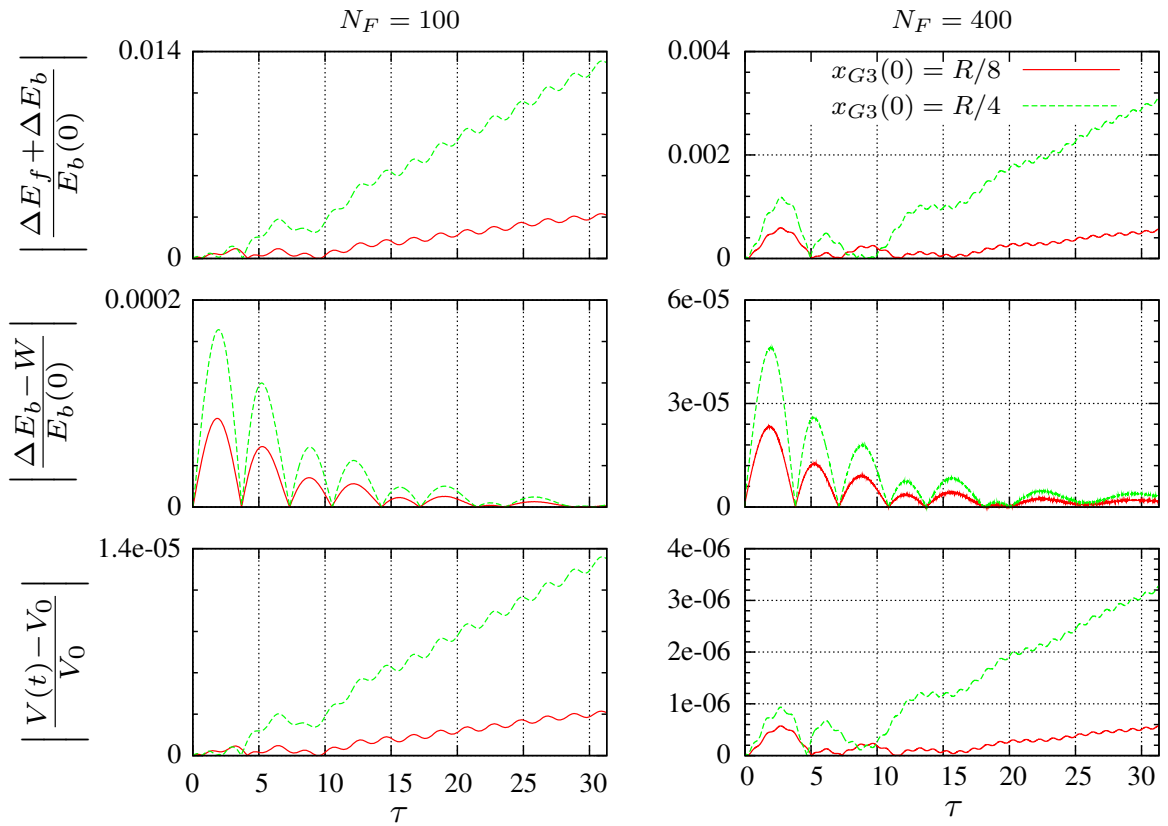

which was not the case using a fixed time step. However, it appears that the energy $E_{f}+E_{b}$ keeps increasing in absolute value after the end of the free decay with the second-order Taylor explicit time stepping. This error is more important for large amplitude.

For this particular case, it has been found that using a symplectic-like first-order integrator as defined in Sect. 2.2.1 leads to a much better conservation of the energy, as it can be seen in Fig. 15 for the initial release of the cylinder at $x_{G 3}(0)=R / 4$.
Moreover, we clearly see in Fig. 15 that the variation reaches a limit which is not the case for the explicit secondorder Taylor series.

\section{Response amplitude operators of a 2D rectangular barge}

Finally, we propose validating our model against the more demanding test case of a $2 \mathrm{D}$ rectangular barge subjected to 
Fig. 15 Freely floating cylinder of radius $R$ : energy $(l e f t)$ and volume (right) conservation for initial amplitude $R / 4$ with two numerical schemes for the finest mesh of parameter $N_{F}=400$

Fig. 16 Rectangular barge in waves of parameters $H=1 \mathrm{~cm}$ and $H=5 \mathrm{~cm}$ : first-order response amplitude operator for sway motion $\frac{x_{G 1}}{A}$ with respect to the non-dimensional frequency $\xi=\omega^{2} \frac{B}{2 g}$

Fig. 17 Rectangular barge in waves of parameters $H=1 \mathrm{~cm}$ and $H=5 \mathrm{~cm}$ : first-order response amplitude operator for heave motion $\frac{x_{G 3}}{A}$ with respect to the non-dimensional frequency $\xi=\omega^{2} \frac{B}{2 g}$
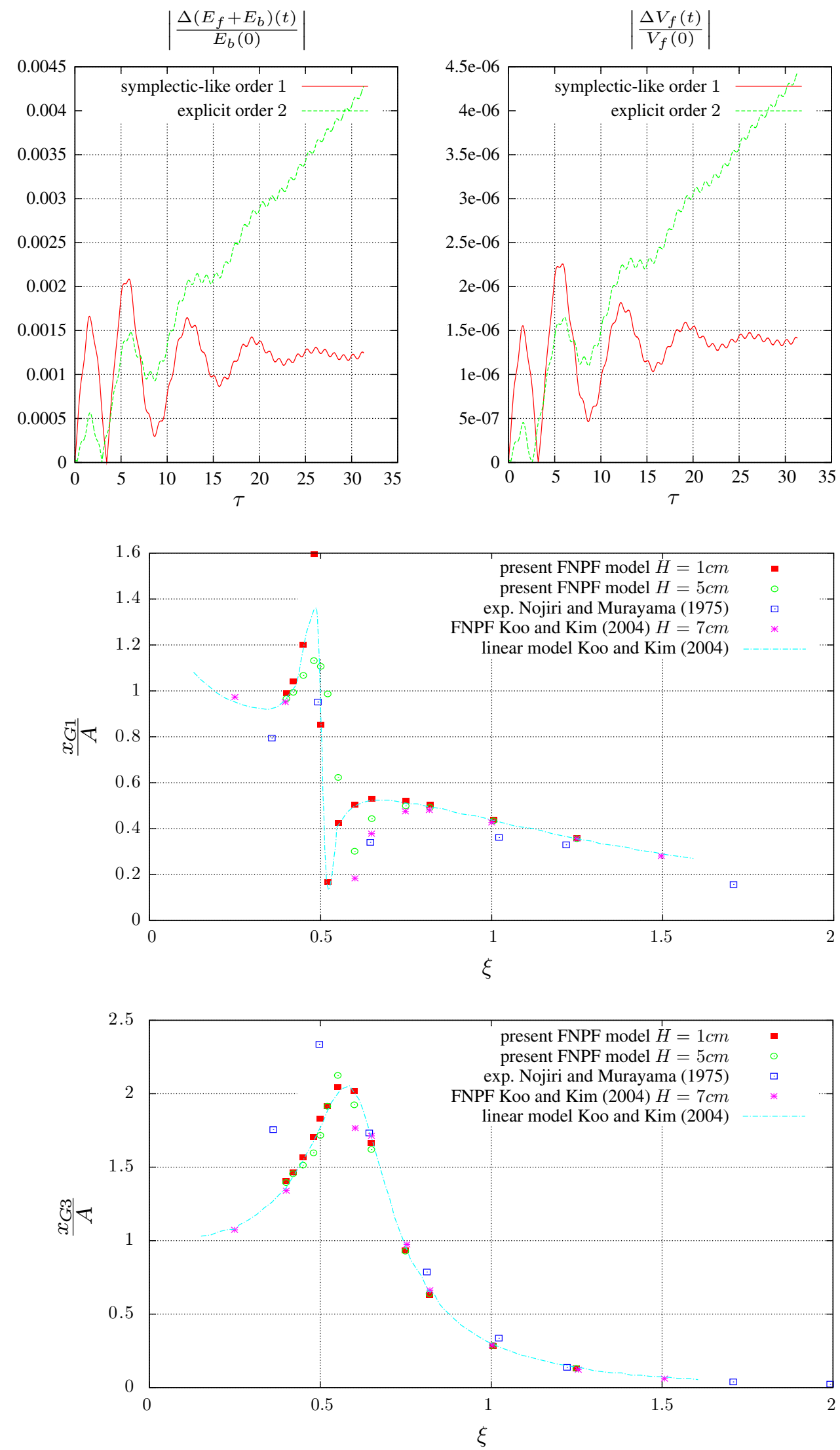
Fig. 18 Rectangular barge in waves of parameters $H=1 \mathrm{~cm}$ and $H=5 \mathrm{~cm}$ : first-order response amplitude operator for roll motion $\frac{x_{G 3}}{\frac{\omega^{2}}{g} A}$ with respect to the non-dimensional frequency $\xi=\omega^{2} \frac{B}{2 g}$

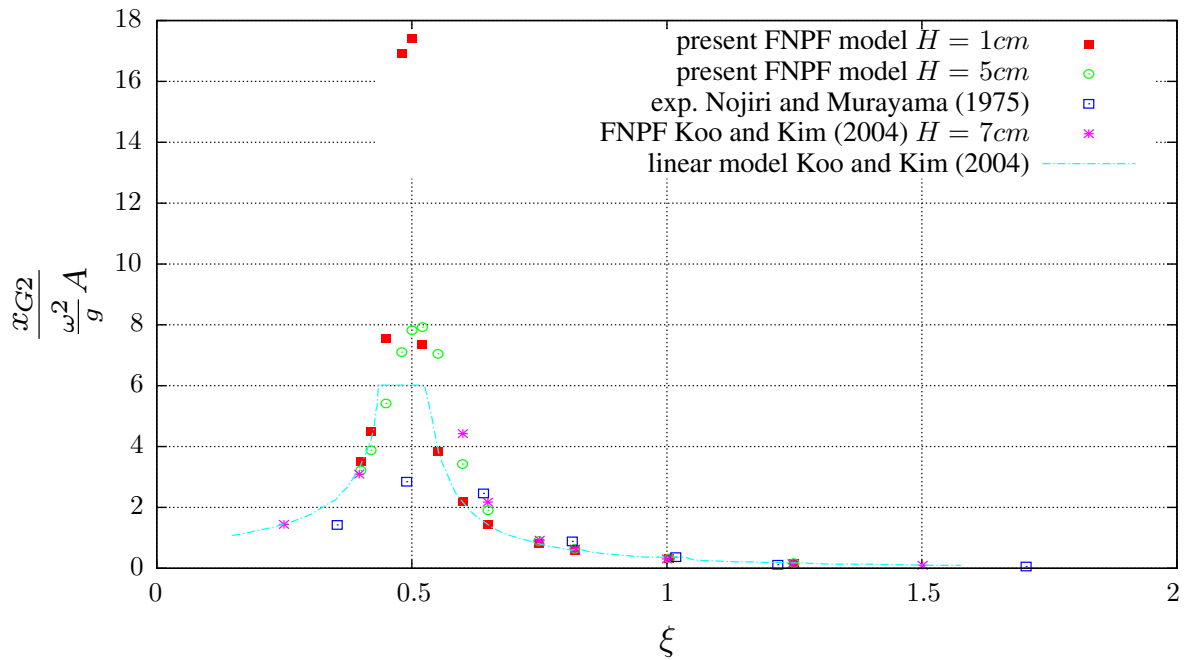

incident waves of various amplitudes. This test case has been used, e.g., by Koo and Kim (2004). We model a 2D rectangular barge with rounded off corners of radius $r=0.064 \mathrm{~m}$, of width $B=0.5 \mathrm{~m}$ and of initial draft $T=0.25 \mathrm{~m}$. The body mass is $M=125 \mathrm{~kg}$ and moment of inertia $I=4.05 \mathrm{~kg} \mathrm{~m}^{2}$. The motion in the $x$ direction is constrained with a spring of stiffness $K=197.58 \mathrm{~N} \mathrm{~m}^{-1}$ and a damping coefficient of $C=19.8 \mathrm{~N} \mathrm{~m}^{-1} \mathrm{~s}^{-1}$. The keel to center of gravity distance is $\mathrm{KG}=0.135 \mathrm{~m}$. We use a wave tank of length $L_{x}=10 \lambda$ for each considered wave. In the model, we specify as a leftward boundary condition an incident linear Airy wave profile of normal particle velocity $\phi_{n}$ and local particle acceleration $\phi_{\text {tn }}$ and additionally use an active absorbing beach in front of the wave generation area. The discretization in results presented below has $N_{f}=60$ nodes per dominant wavelength. We compute two set of frequencies for a small wave height $H=1 \mathrm{~cm}$ and a moderate one $H=5 \mathrm{~cm}$. Our results are compared : (i) to the fully non-linear computations of Koo and Kim (2004) obtained with a similar FNPF model for $H=7 \mathrm{~cm}$; (ii) to the experimental results of Nojiri and Murayama (1975) digitized from the paper of Koo and Kim (2004); and (iii) to the linear computations of Koo and Kim (2004). The first-order response amplitude operators for each mode of motion are represented in Figs. 16, 17, 18. The comparison between the small amplitude wave case and the linear computations of Koo and Kim (2004) is quite satisfactory. It is interesting to note that the first-order response obtained with the wave height $H=5 \mathrm{~cm}$ significantly deviates from that obtained with $H=1 \mathrm{~cm}$ near the resonance frequency range.

\section{Conclusions}

We presented computations of the interactions of a floating body with a free surface flow modeled by the fully non- linear potential flow (FNPF) theory. The present FNPF model is implemented based on a higher-order boundary element method (BEM). Although these results are being extended to three dimensions, the present applications are only for twodimensional cases. In this paper, we extended the implicit coupling scheme of Guerber et al. (2012), which was limited to submerged bodies, to surface-piercing rigid bodies. Since there are more reference solutions in the literature for this case, we first verified the accuracy and convergence of our scheme, for the forced motion (heaving and swaying) of a rigid body on the free surface. Moreover, we also demonstrated the advantage, in terms of energy and volume conservation, of using a time stepping inspired by the symplectic integrators. In particular, besides global errors such as on mass and energy conservation, we assessed the accuracy of the computed hydrodynamic force applied to the body as a result of its motion, which is key to accurately solving cases with a freely floating body. In all cases, for small amplitude body motion, we observed a very good agreement with results from the linear theory. In such cases, the conservation of total fluid volume is achieved within a high degree of accuracy. In general, to assess the convergence of various model results, we computed a $L^{2}$ error norm of results in various boundary discretizations, compared to those obtained in the finest discretization. This norm was computed at a set of internal points within the fluid domain, which are kept at a fixed position during the simulations. In all cases, convergence of the selected field variables, in terms of the $L^{2}$ error norm, shows a good behavior both for forced and freely floating body motion. For large amplitude motion cases, the $L^{2}$ error norm of the variables has a similar behavior with respect to the discretization, but the smallest errors achieved are larger than for small amplitude motion. We believe that, for such cases, the current geometric updating of the free surface intersection nodes with the body and the regridding of the free surface discretization that results, may not be sufficient for achieving 
a high volume conservation. For freely floating bodies, the convergence of the algorithm is studied for various amplitudes of motion in free decay tests. We find that the implicit method of van Daalen (1993) and Tanizawa (2000) gives a satisfactory agreement with the analytical results of Maskell and Ursell (1970). A larger discrepancy, however, is found for large amplitude free decay motions, which indicates the need for developing a way to include viscous effects in the present FNPF model. A preliminar investigation of the capability of the model to predict the free motion of a floating body in waves is done and a fine agreement with other references is found.

In future work, besides extending the present approach to the 3D-FNPF model of Grilli et al. (2001), we will refine the treatment of intersection points between the rigid body and the free surface and study cases both in 2D and 3D when body motions are forced by waves generated in the model (both regular and irregular). We also aim to generalize to higher order, the present first-order symplectic-like integrator.

Acknowledgments This work was partly funded by the French Research Agency (CIFRE agreement \# 2011-1724). Stephan Grilli acknowledges support for this work from Grant N000141310687 of the US Office of Naval Research.

\section{References}

Andersen P, Wuzhou H (1985) On the calculation of two-dimensional added mass and damping coefficients by simple Green's function technique. Ocean Eng 12(5):425-451

Bai W, Eatock Taylor R (2006) Higher-order boundary element simulation of fully nonlinear wave radiation by oscillating vertical cylinders. Appl Ocean Res 28(4):247-265

Bai W, Eatock Taylor R (2009) Fully nonlinear simulation of wave interaction with fixed and floating flared structures. Ocean Eng 36(3):223-236

Berkvens P (1998) Floating bodies interacting with water waves: Development of a time-domain panel method, $\mathrm{PhD}$ thesis, Universiteit Twente, The Netherlands

Bonnet M (1995) Equations intégrales et éléments de frontiere (in French), CNRS Editions/Eyrolles

Cointe R (1989) Quelques aspects de la simulation numérique dun canal à houle (284 pages in French), $\mathrm{PhD}$ thesis, Ecole Nationale des Ponts et Chaussées, Paris, France

Dias F, Bridges T (2006) The numerical computation of freely propagating time-dependent irrotational water waves. Fluid Dyn Res 38(12):803-830

Dommermuth D, Yue D (1987) Numerical simulations of nonlinear axisymmetric flows with a free surface. J Fluid Mech 178:195219

Frank W (1967) Oscillation of cylinders in or below the free surface of deep fluids. DTIC Document, Report 2375. Naval Ship Research and Development Center, Hydromechanics Laboratory, Washington, D.C., USA

Ghasemi A, Pathak A, Raessi M (2014) Computational simulation of the interactions between moving rigid bodies and incompressible two-fluid flows. Comput Fluids 94:1-13
Grilli S, Guyenne P, Dias F (2001) A fully non-linear model for threedimensional overturning waves over an arbitrary bottom. Int J Numer Methods Fluids 35(7):829-867

Grilli S, Skourup J, Svendsen I (1989) An efficient boundary element method for nonlinear water waves. Eng Anal Bound Elements 6(2):97-107

Grilli S, Subramanya R (1993) A computer program for transient wave run-up. Research Report No. CACR-93-02. University of Rhode Island, Kingston, RI, USA

Grilli S, Subramanya R (1996) Numerical modeling of wave breaking induced by fixed or moving boundaries. Comput Mech 17(6):374391

Grilli S, Svendsen I (1990) Corner problems and global accuracy in the boundary element solution of nonlinear wave flows. Eng Anal Bound Elements 7(4):178-195

Guerber E, Benoit M, Grilli S, Buvat C (2012) A fully nonlinear implicit model for wave interactions with submerged structures in forced or free motion. Eng Anal Bound Elements 36(7):1151-1163

Hadžić I, Hennig J, Perić M, Xing-Kaeding Y (2005) Computation of flow-induced motion of floating bodies. Appl Math Model 29(12):1196-1210

Ito $S$ (1977) Study of the transient heave oscillation of a floating cylinder, $\mathrm{PhD}$ thesis, Massachusetts Institute of Technology

Kane C (1999) Variational integrators and the Newmark algorithm for conservative and dissipative mechanical systems, $\mathrm{PhD}$ thesis, $\mathrm{Cal}-$ tech

Kent C (2005) A pseudo-spectral method for calculating wave-body interaction using an explicit free-surface formulation, $\mathrm{PhD}$ thesis, University of Michigan

Koo W, Kim M (2004) Freely floating-body simulation by a 2D fully nonlinear numerical wave tank. Ocean Eng 31(16):2011-2046

Koo W, Kim M (2007) Fully nonlinear wave-body interactions with surface-piercing bodies. Ocean Eng 34(7):1000-1012

Lee C (1968) The second-order theory of heaving cylinders in a free surface. J Ship Res 12(4):313-327

Lew A, Marsden J, Ortiz M, West M (2004) Variational time integrators. Int J Numer Methods Eng 60(1):153-212

Li Y, Lin M (2012) Regular and irregular wave impacts on floating body. Ocean Eng 42:93-101

Liu Y, Xue M, Yue D (2001) Computations of fully nonlinear threedimensional wave-wave and wave-body interactions, part 2: nonlinear waves and forces on a body. J Fluid Mech 438:41-66

Ma Q, Yan S (2009) QALE-FEM for numerical modelling of non-linear interaction between 3D moored floating bodies and steep waves. Int J Numer Methods Eng 78(6):713-756

Maskell S, Ursell F (1970) The transient motion of a floating body. J Fluid Mech 44(2):303-313

Nojiri N, Murayama K (1975) A study on the drift force on two dimensional floating body in regular waves. Trans West Jpn Soc Nav Arch 51:131-152

Porter W (1960) Pressure distributions, added-mass, and damping coefficients for cylinders oscillating in a free surface. Technical report, DTIC Document

Rahman M, Mizutani N, Kawasaki K (2006) Numerical modeling of dynamic responses and mooring forces of submerged floating breakwater. Coast Eng 53(10):799-815

Tang H, Huang C, Chen W (2011) Dynamics of dual pontoon floating structure for cage aquaculture in a two-dimensional numerical wave tank. J Fluids Struct 27(7):918-936

Tanizawa K (1995) A nonlinear simulation method of 3D body motions in waves. J Soc Nav Arch Jpn 178:179-191

Tanizawa K (2000) The state of the art on numerical wave tank. In: Proceedings of 4 th Osaka colloquium on seakeeping performance of ships, pp 95-114 
Tasai F, Koterayama W (1976) Nonlinear hydrodynamic forces acting on cylinders heaving on the surface of a fluid. Rep Res Inst Appl Mech Kyushu Univ 24:1-39

van Daalen E (1993) Numerical and theoretical studies of water waves and floating bodies, PhD thesis, Universiteit Twente, The Netherlands

Vugts JH (1968) The hydrodynamic coefficients for swaying, heaving and rolling cylinders in a free surface. In: Report // Nederlands Scheepsstudiecentrum TNO, vol 112. Netherlands Ship Research Centre TNO, Shipbuilding Department

Wang Q (2005) Unstructured MEL modelling of nonlinear unsteady ship waves. J Comput Phys 210(1):368-385
Wehausen J (1971) The motion of floating bodies. Annu Rev Fluid Mech 3(1):237-268

Yamashita S (1977) Calculations of the hydrodynamic forces acting upon thin cylinders oscillating vertically with large amplitude. J Soc Nav Arch 141:61-70

Yan S, Ma Q (2007) Numerical simulation of fully nonlinear interaction between steep waves and 2D floating bodies using the QALE-FEM method. J Comput Phys 221(2):666-692

Zhou B, Ning D, Teng B, Bai W (2013) Numerical investigation of wave radiation by a vertical cylinder using a fully nonlinear HOBEM. Ocean Eng 70:1-13 